\title{
Elastic-decohesive constitutive model for sea ice
}

\author{
H. L. Schreyer, ${ }^{1}$ D. L. Sulsky, ${ }^{2}$ L. B. Munday, ${ }^{1}$ M. D. Coon, ${ }^{3}$ and R. Kwok ${ }^{4}$ \\ Received 30 September 2005; revised 31 December 2005; accepted 26 June 2006; published 24 October 2006.
}

[1] One of the dominant characteristics of Arctic ice is the development and persistence of leads. Leads are critically important for climate modeling since they are a source for new ice and an avenue for heat exchange between the atmosphere and ocean. Therefore a specific representation for leads in a constitutive model for pack ice is considered essential. Presented here is an elastic-decohesive constitutive model whose purpose is to specifically indicate when a lead is initiated, to provide the orientation of the lead, to give the mode of failure, and to allow for the numerical determination of the width of leads. Both laboratory and in situ data of sea ice provide the motivation for the basic structure of the model. Sample paths in stress and strain space are used to illustrate how the model can simulate failure. The model can predict multiple failure planes, a feature that is necessary to simulate crack branching. Preexisting planes of weakness such as those formed from previous leads can also be accommodated.

Citation: Schreyer, H. L., D. L. Sulsky, L. B. Munday, M. D. Coon, and R. Kwok (2006), Elastic-decohesive constitutive model for sea ice, J. Geophys. Res., 111, C11S26, doi:10.1029/2005JC003334.

\section{Introduction}

\subsection{Background}

[2] Within the Arctic sea-ice, stresses are formed from wind, ocean currents and other sources. These stresses are continuously changing and cause the opening and closing of cracks (leads) in the pack ice that may be thousands of kilometers in length. Large and small-scale photographs of Arctic ice reveal the system of open leads. Leads are important for climate modeling because an open lead provides an avenue for heat transfer from the ocean to the atmosphere. The formation of new ice within leads upon refreezing is also noteworthy because of the large amounts of energy required to create ice and of brine injected into the ocean. Another obvious feature of the Arctic landscape is ridges formed when leads are forced to close, crushing new ice within the lead.

[3] One of the first attempts to provide a continuum constitutive model for representing the composite effect of the ice sheet with leads and ridges was that of Coon et al. [1974] based on a two-dimensional elastic-plastic solid with an associated flow rule. Ridging was included in the approach with parameters chosen to provide an overall energy balance [Rothrock, 1975] and evolution equations were added for thickness distribution [Thorndike et al., 1975]. It was envisioned that this model would be used in numerical simulations where the resolved length scale was hundreds of kilometers. On this scale there are many leads

\footnotetext{
${ }^{1}$ Department of Mechanical Engineering, University of New Mexico, Albuquerque, New Mexico, USA.

${ }^{2}$ Department of Mathematics and Statistics, University of New Mexico, Albuquerque, New Mexico, USA.

${ }^{3}$ Northwest Research Associates, Seattle, Washington, USA.

${ }^{4}$ Jet Propulsion Laboratory, Pasadena, California, USA.
}

Copyright 2006 by the American Geophysical Union. 0148-0227/06/2005JC003334\$09.00 within a computational element and their effect could be modeled using an isotropic model with a compressive failure strength associated with ridge formation. The basic model was enhanced to reflect observed anisotropic features when failure occurs [Coon et al., 1998].

[4] In the same vein, Hibler [1979] introduced a viscousplastic isotropic model for ice. Implicitly, the model contains history parameters, the distribution of ice thicknesses that evolve according to predictions of dilation or compaction. Models of this type generally give an indirect measure of lead opening through an integration of the divergence of velocity, and infer the direction of leads through plots of divergence over the spatial domain. However, these models provide a computationally efficient scheme to predict the motion of Arctic ice as well as an indication of the area of open water and the amount of new ice created over a winter season. For the original purpose, these models work admirably well. Various modifications have been proposed [Hibler and Schulson, 1997; Hibler, 2001] including one in which nonphysical elasticity was introduced to enhance further the computational speed [Hunke and Dukowicz, 1997; Hunke, 2001]. The models with a basic viscousplastic feature have been primarily isotropic. However Hibler and Schulson [2000] and Wilchinsky and Feltham [2004] have proposed anisotropic versions to reflect better the oriented features associated with leads.

[5] In contrast to the preceding models, Hopkins [1996, 2001] discards the continuum hypothesis and uses an approach based on discrete elements with frictional and elastic-plastic flow laws between adjacent floes. The overall constitutive behavior is connected intimately with the assumed forms for the interactions between floes. Although this model might be entirely appropriate when the pack breaks up with warm weather, there seems to be a question as to whether or not it can represent pack ice during the 
winter when the observed number and orientation of active leads is limited.

[6] As computers increase in power, it is possible to consider simulations of the Arctic with a computational cell size on the order of tens of kilometers. At this scale there no longer are many leads in a computational cell and a model needs to be developed that accounts for individual leads. It is believed that a more precise constitutive equation can bring significant improvements to detailed predictions of the formation of leads and new ice and, consequently, corresponding improvements in the prediction of ice motion and deformation. The proposed approach is to model the formation of a lead specifically through the use of a constitutive equation that relates traction at a point on a material surface to the amount of discontinuity in displacement. Such models are referred to variously as discrete, discontinuum, cohesive crack and decohesive constitutive equations, of which the latter terminology will be used in this text. Decohesion is defined to be the loss of strength that occurs with an increase in a suitable norm of the displacement discontinuity. Comprehensive versions of such models provide the stress at which a crack initiates, the orientation of the crack, the mode at which failure occurs, and the criterion for which all traction-carrying capability is lost. Classical models such as those of Rankine, Tresca and Mohr-Coulomb provide some but not all of these properties.

[7] Because of the importance of leads in simulating both the dynamic and thermodynamic features of the Arctic ice pack, the use of a decohesive constitutive equation is warranted in an attempt to model explicitly the initiation and evolution of leads. Schulson [2004] argues persuasively that features exhibited by failure of Arctic ice are similar to those shown by failure of experimental specimens and that compressive failure of ice and rocks have similar features [Renshaw and Schulson, 2001]. In fact, the similarity is much more extensive since the failure modes for ice described by Sammonds et al. [1998] are comparable to those of concrete. Accordingly, aspects of the model and some terminology are borrowed from these other fields.

\subsection{Motivation}

[8] Since there are several models of ice in the literature, there must be a compelling reason to introduce the new one that is the focus of this paper. The first point to be emphasized is that decohesion is widely used in other fields [Planas et al., 2003].

[9] Second, from the figures provided by Kwok [1998] based on RGPS data, one cannot help but notice the existence of long linear features that might reasonably be interpreted as large active leads. Therefore, it would seem that an attempt to explicitly include a constitutive formulation for predicting the creation of leads ought to be met with some enthusiasm. The alternative is to infer the presence of a lead based on constitutive equations formulated under the questionable assumption of displacement continuity or compatibility.

[10] Third, current popular models used for basin-wide simulations may not be appropriate for sea ice at scales of $10 \mathrm{~km}$ or less. It is yet to be seen if detailed features of sea ice motion impact larger scale flow.
[11] In addition to their effects on climate, being able to explicitly predict the creation of a lead on the scale of 10 kilometers, say, could be of great benefit in determining the susceptibility of fish and other wildlife to a largescale release of contaminants near a coast because such substances often end up in leads. The sudden creation of a lead, itself, near the coast may endanger humans who may be inadvertently caught offshore. The motion of ice is also important for determining the susceptibility of offshore structures to damage, or the passage of ice into the Atlantic. It seems plausible that a more accurate representation of the state of stress in the presence of leads, everything else being equal, should result in a higher likelihood of accurately predicting the motion of ice at scales of the order of widths of leads. For example, a model specifically designed to represent leads can allow for a normal stress parallel to the lead whereas a representation of a lead based on some continuum models results in all components of stress being zero. Furthermore, over the course of a season, many regions of the ice cover undergo large deformations, so if history variables such as those necessary to describe leads, are to be accurately tracked a suitable numerical scheme such as that described by Sulsky et al. [2006] must be used concurrently with an improved constitutive equation.

[12] Long-term climate predictions are coupled thermodynamically with the thickness distribution of ice. It is not clear that the existing models would predict the same amount of ice production based on divergence. Koerner [1973] and Kwok [2006] have estimated that 20-30\% of the sea ice added each year in the permanent ice zone is a result of freezing within leads. In the seasonal ice zone, the percentage is even higher. Therefore, it seems plausible to assume that as climate models improve and numerical algorithms become more efficient, it would make sense to incorporate models that provide explicit representations for widths of leads.

[13] A problem with many existing decohesion models is that they are studied as academic exercises and often do not reflect features shown by experimental data. The proposed decohesion model is an attempt to reflect the essential fracture characteristics of ice without the overwhelming detail that would be impossible to handle numerically. Of these characteristics, it is believed that the stress at which failure initiates, the orientation of failure, and the fracture energy represent the essential features. With the assumption that the effect of a crack can be smeared over an element, the result is a constitutive numerical algorithm that is both efficient and that captures the induced anisotropy associated with failure.

\subsection{Outline of Paper}

[14] Observations based on experimental data from Schulson [2001] are described briefly in the first part of the next section. Then the limitations of classical models are provided to motivate the need for the proposed decohesive model which is presented next. The model is phenomenological and relatively simple since applications to largescale numerical simulations represent the intended use. Nevertheless, there is an attempt to reflect the essential aspects of both experimental data and micro-structural properties within the formulation. The primary focus is on predicting correctly the stress at which a lead initiates or 


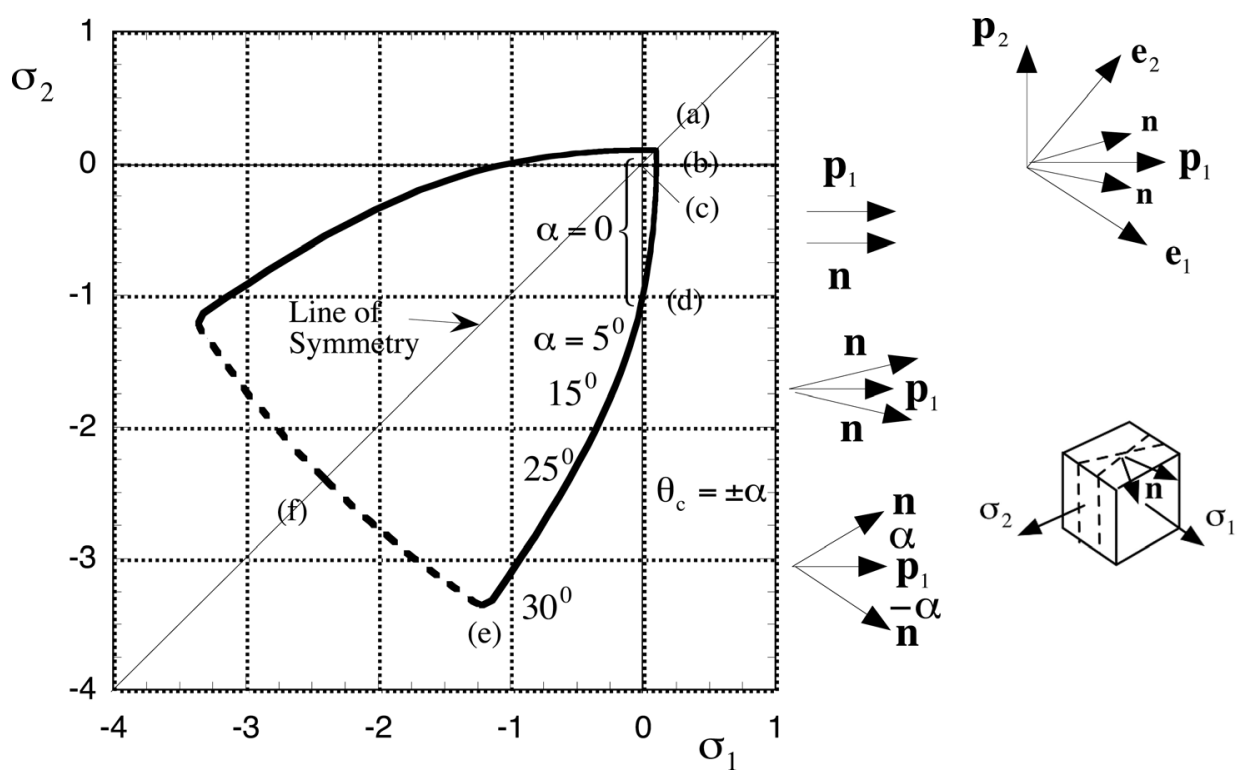

Figure 1. Generic aspects of a failure surface in plane stress and the orientation of the surface of material cracking (failure).

re-activates, and the orientation of the lead. Significant modifications from laboratory-based material parameters are necessary for large-scale simulations, and this point is also discussed.

[15] Section 3 describes how leads open once failure is detected and initiated. Then, based on the assumption that the continuum deformation of Arctic ice is sufficiently small so that elasticity can be invoked, Section 4 provides an explanation of how decohesion can be combined with elasticity to obtain a constitutive equation subroutine for use in large-scale numerical simulations. The implications of the elastic-decohesion model are shown through examples for selected stress and strain paths. An illustrative example indicates how an initial oriented weakness can be reflected in the model. In addition, it is demonstrated that two or more planes of failure can be predicted at a point, a feature that allows for the potential of predicting crack branching in large-scale simulations.

[16] A slight addition to the elastic-decohesion model provides a technique for modeling the closure and shear of a frozen lead as described in section 5. Finally, the summary emphasizes those aspects of the proposed model that are not being included in current constitutive equations used to represent Arctic ice.

\section{Experimental Data and Classical Models for Failure}

\subsection{Essential Aspects of Failure in Plane Stress}

[17] Schulson [2001] provides a comprehensive experimental study of the failure of specimens formed from both fresh and salt water, and the study includes the effects of strain rate and temperature on strength and mode of failure. Although in situ ice is generally considered to be weaker than laboratory ice, and in concurrence with the convincing arguments of Schulson [2004], it is believed that modes of failure for relatively small specimens are similar to those observed on a large scale. Schulson is a strong advocate for using the Mohr-Coulomb model; here it is argued that a different approach is even better. The generic features of these experimental data are used to motivate a particular form of the proposed constitutive equation. The goal is to get the correct qualitative behavior for applications to Arctic ice, and not to provide a constitutive equation suitable for modeling ice formed in a laboratory.

[18] Let $\boldsymbol{\sigma}$ denote the stress tensor with components $\sigma_{11}$, $\sigma_{22}$ and $\sigma_{12}$ for an orthonormal basis $\mathbf{e}_{1}$ and $\mathbf{e}_{2}$ in the plane of the ice sheet. The principal directions of stress, $\mathbf{p}_{1}$ and $\mathbf{p}_{2}$, also form an orthonormal basis associated with principal values $\sigma_{1}$ and $\sigma_{2}$. Failure in plane stress is often described by a failure surface in terms of principal stresses. A generic failure surface representative of laboratory ice as indicated by the data of Schulson is sketched in Figure 1. The figure depicts the failure surface in stress space. That is, if ice is loaded from zero stress with $\sigma_{1}$ proportional to $\sigma_{2}$, failure will occur when $\sigma_{1}$ and $\sigma_{2}$ reach the values indicated on the failure surface. Failure in equal biaxial tension is identified as point (a), failure in uniaxial tension by point (b), failure in pure shear at (c) (one principal value of stress is the negative of the other), while failure in uniaxial compression is identified as (d). The value of the uniaxial compressive stress is typically an order of magnitude larger than the failure stress in uniaxial tension. As the stress state is extended into the regime where both principal stresses are compressive, the failure surface extends from (d) to a maximum at point (e). The remaining regime for compressive stresses is given as the segment of the curve shown as a dotted line from (e) to equal biaxial compression at point (f). With the assumption of initial isotropy in the plane, the remainder of the curve is merely a reflection about the symmetry line $(\mathrm{a}-\mathrm{f})$.

[19] Schulson also provides the orientation of the normal to the plane of material failure for states of stress at failure. Again, only the generic features of this aspect of the data will be summarized. As indicated in Figure 1, suppose a unit normal vector $\mathbf{n}$ lies within the plane of stress, and 
forms an angle, $\theta$, with respect to the first principal direction $\mathbf{p}_{1}$. Define the normal vector to the surface of material failure by the critical angle, $\theta_{c}$. For the regime (a), (b), (c) $-(\mathrm{d}), \mathbf{n}$ is equal to $\mathbf{p}_{1}$, the direction of maximum principal stress, or $\theta_{c}=0$. Such an orientation is typically associated with brittle materials. In particular, if $\theta_{c}=0$ for uniaxial compression, the resulting feature is called axial splitting and is seen in some experimental specimens formed from concrete. However, in the regime (d)-(e), two orientations of failure exist $\theta_{c}= \pm \alpha$ with $\alpha$ increasing smoothly from zero at (d) to a maximum of about $30^{\circ}$ at (e). Finally, for the dotted segment of the failure curve, the normal is out of the plane and, again, is in the direction of maximum principal stress which is zero (from a three-dimensional viewpoint) with the other two principal stresses in the plane being negative.

[20] For pack ice, failure where the normal has a component out of the plane is rarely, if ever, observed. Although, failure stresses and the shape of the failure curves will depend on factors such as salinity, temperature and strain rate, it is not the purpose of this paper to provide a detailed model that captures these effects. Instead the focus is to provide a model that provides the essential features of the solid line in Figure 1 for both the stress at failure and the orientation of the surface of material failure.

\subsection{Classical Models of Failure}

[21] In order to describe various models in a succinct manner, and for future use, a standard notation is now introduced. Small deformations are assumed so there is no need to differentiate among the various stress and strain tensors. The traction on any surface with normal $\mathbf{n}$ is

$$
\tau=\boldsymbol{\sigma} \cdot \mathbf{n}
$$

Introduce another orthonormal pair of base vectors consisting of $\mathbf{n}$ and $\mathbf{t}$, normal and tangential unit vectors to the plane of material failure. Then $\tau_{n}=\sigma_{n n}$ and $\tau_{t}=\sigma_{n t}$ are the normal and shear components of traction, respectively, and the remaining component of stress is $\sigma_{t t}$.

[22] In order to describe the classical models, it is assumed that failure on a material surface with given orientation, $\mathbf{n}$, can be described through the use of a decohesion function, $F_{n}^{D}$, which depends on material properties and the traction vector. The latter dependence implies that the function depends on the orientation of the particular material surface being considered. The function is constructed so that $F_{n}^{D}<0$ indicates failure is not occurring, $F_{n}^{D}=0$ identifies the onset of failure, and $F_{n}^{D}>0$ is not allowed. To determine if failure is initiated at a point, all possible orientations must be considered with the critical direction being the one that maximizes $F_{n}^{D}$.

[23] As described for Figure 1, a point on the decohesive surface, $F_{n}^{D}=0$, can be obtained by incrementing radially from the origin in stress space. Each point on the radial path defines a state of stress which must be evaluated to determine if decohesion has occurred. A search for the critical orientation of $\mathbf{n}$ is performed by maximizing $F_{n}^{D}$ with respect to the angle $\theta$ where

$$
\mathbf{n}=\cos \theta \mathbf{p}_{1}+\sin \theta \mathbf{p}_{2} \quad 0 \leq \theta<\pi
$$

Once the stress path has been extended so that the maximum value of $F_{n}^{D}$ is zero, one point on the decohesion surface has been defined. The unit vector $\mathbf{n}$ defined by the corresponding critical value, $\theta=\theta_{c}$, provides the orientation of the physical surface where a crack initiates. The process is repeated for different radial paths until the complete decohesion surface is obtained.

[24] The classical criteria for failure are simple enough so that analytical expressions are available for both the decohesion surface and the orientation of crack initiation. However, the general approach described above provides a common framework for both classical models and the alternative formulation that will be introduced later.

[25] In summary, a classical decohesion function, $F^{D}$, is defined as follows:

$$
F^{D}=\max _{\forall \mathbf{n}} F_{n}^{D} \quad F_{n}^{D} \equiv F_{n}^{D}(\boldsymbol{\tau})
$$

with failure indicated when $\boldsymbol{F}^{D}=0$. The decohesion functions of Rankine, $F_{n}^{R}$, and Tresca, $F_{n}^{T}$, each depend on only one component of the traction, $\tau$, as follows:

$$
F_{n}^{R}=\frac{\tau_{n}}{\tau_{n f}^{R}}-1 \quad F_{n}^{T}=\frac{\tau_{t}^{2}}{\left(\tau_{s f}^{T}\right)^{2}}-1
$$

The material parameters $\tau_{n f}^{R}$ and $\tau_{s f}^{T}$ are the critical values of normal and shear traction, respectively, and are associated with these specific criteria. The surface $F^{R}=0$ denotes the maximum tensile stress criterion of Rankine and is shown in Figure 2a for plane stress in terms of principal components. The critical orientations for the surfaces of material failure are unique and these directions are also given $\left(\theta_{c}=0\right.$ for plane stress). Similarly, the surface $F^{T}=0$, shown in Figure $2 b$, is the maximum shear-stress criterion of Tresca. However, now for each point of failure there are two possible orientations of material surface whereby $\mathbf{n}$ makes equal angles with the direction of maximum principal stress and $\theta_{c}= \pm 45^{\circ}$. The two possible surfaces of material failure are orthogonal to each other. On the dotted segment of the surface, the normal does not lie in the plane of stress.

[26] Another criterion, which is often used for geological materials, is that of Mohr-Coulomb which involves both the normal and shear components of traction as follows:

$$
F_{n}^{M C}=\frac{\left|\tau_{t}\right|}{\tau_{s f}^{M C}}+\frac{\tau_{n}}{\tau_{n f}^{M C}}-1
$$

The essential idea is a generalization of the Tresca criterion in that failure in shear on a given surface is assumed to depend on the amount of normal traction. If $\tau_{n f}^{M C}$ is infinite, the criterion is that of Tresca; if $\tau_{s f}^{M C}$ is infinite, the result is Rankine's criterion. Similarly to the Tresca criterion, there are two possible orientations for material failure but, instead of $\theta_{c}= \pm 45^{\circ}$, the normal to the surface of failure is now $\theta_{c}= \pm \alpha$ with respect to the direction of maximum principal stress in the plane of maximum and minimum principal directions of stress and tan $2 \alpha=\tau_{n f}^{M C} / \tau_{s f}^{M C}$. The surface $F^{M C}=0$ is shown in Figure $2 \mathrm{c}$ for plane stress. For example, if $\tau_{s f}^{M C}=1.4 \tau_{n f}^{M C}$ the positive intercept of the $\sigma_{1}$ axis for $\sigma_{2}=0$ is $\sigma_{1}=0.9 \tau_{n f}^{M C}$. Similarly, if $\sigma_{1}=0$, 


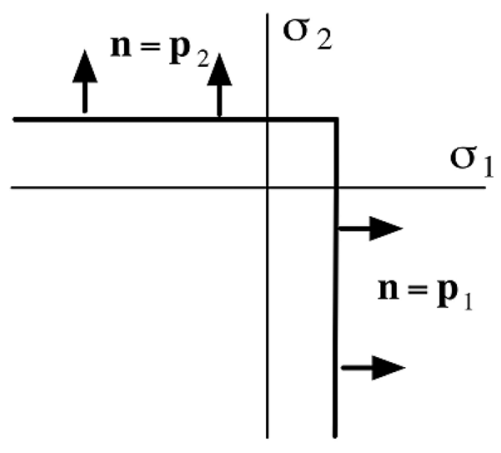

(a) Rankine criterion

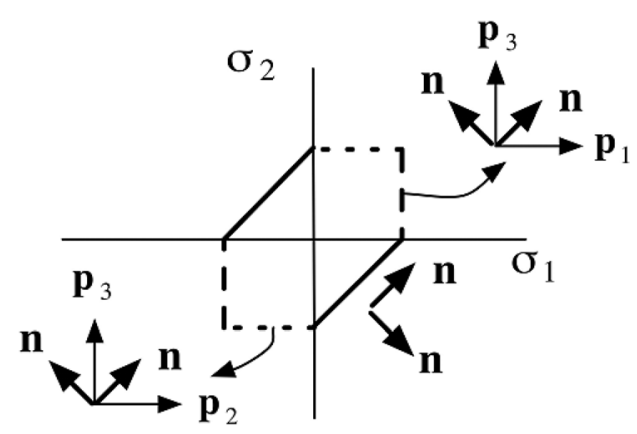

(b) Tresca criterion

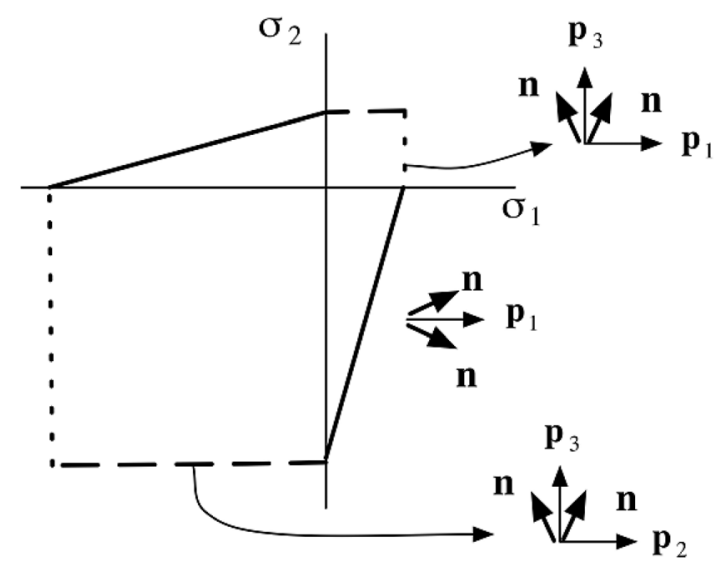

(c) Mohr-Coulomb criterion

Figure 2. Sketches of curves showing failure initiation including orientations of the normal to surfaces of material failure as predicted by classical models. (The normal lies within the plane only for the solid portions of each curve.)

the intercept is $\sigma_{2}=-8.75 \tau_{n f}^{M C}$ which yields a ratio of approximately 10 between the tensile and compressive failure stresses, a ratio often observed for concrete and geological materials. The orientation of the normal of material failure with respect to the direction of maximum principal stress is $\theta_{c}= \pm 18^{\circ}$ for all points of failure. However, the Schulson data, depicted in Figure 1, shows that the orientation of the normal may vary from one point on the failure surface in stress space to another point. The Mohr-Coulomb criterion, which is often used for geological materials, predicts only one value of $\alpha$ with $\theta_{c}= \pm \alpha$ for all states of stress that satisfy the failure criterion. Although reasonable for some of the domain of failure, such an angle is not representative for all states of stress at the initiation of failure. Again, the dotted portion of the surface in Figure 2 indicates that an out-of-plane component exists for the normal vector.

[27] Each criterion has had considerable success in predicting failure, the Rankine criterion for brittle materials, Tresca for ductile materials, and Mohr-Coulomb for concrete and geological materials such as rock and ice. However, as the Schulson data show for ice, and similarly for many other materials, materials often fail according to one criterion for one regime of stress, and to another criterion for a different regime. Specifically, the Rankine criterion is often suitable for tensile states of stress, Mohr-Coulomb for intermediate states, and the Tresca criterion for large compressive states. Not one of these classical criteria has the flexibility to accurately reflect simultaneously both the state of stress at failure and the orientation of the surface of material failure for all paths in plane stress. In particular, none predicts axial splitting. Next, a new model is proposed that attempts to remedy this situation.

\subsection{Proposed Form for Failure Criterion}

[28] A mathematically attractive approach for indicating when failure initiates is one based on loss of ellipticity as indicated by Schreyer [2001]. Such a criterion might be used to suggest when a decohesive model should be implemented. However, it is considerably simpler to just use the value of a decohesive function as an indicator when failure initiates and this is the approach adopted here.

[29] The key new feature in the proposed decohesion function is to allow for the possibility that, in addition to the traction, failure may depend on the component of the stress $\sigma_{t}$, as suggested but not implemented by Planas et al. [2003]. The decohesion function is similar to the Mohr- 


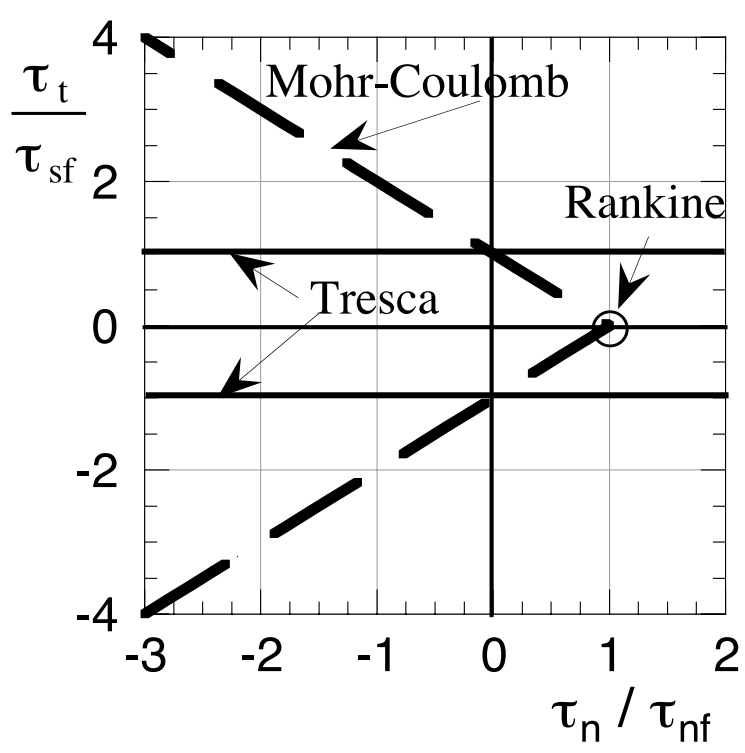

(a) Classical surfaces.

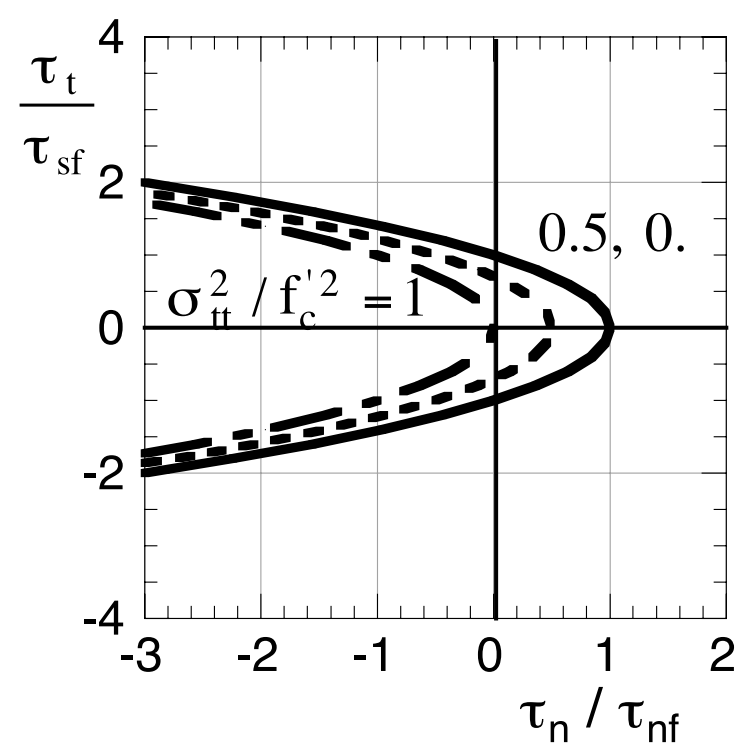

(b) Surfaces based on proposed criterion.

Figure 3. Plots of failure surfaces in $\tau_{n}-\tau_{\mathrm{t}}$ space.

Coulomb function in that both the shear and normal components of the traction are included. The shear and in-plane normal components of stress are included as quadratic terms as follows:

$$
F=\max _{\forall \mathbf{n}} F_{n}\left(\boldsymbol{\tau}, \sigma_{t t}\right) \quad F_{n}=\frac{\tau_{t}^{2}}{\tau_{s f}^{2}}+\frac{\tau_{n}}{\tau_{n f}}+\frac{\sigma_{t t}^{2}}{f_{c}^{\prime 2}}-1
$$

The failure surface is defined by $F=0$. The parameter $\tau_{s f}$ is the shear failure strength when $\tau_{n}=0$ and $\sigma_{\mathrm{tt}}=0$ and must be interpreted differently from the corresponding parameters in the Tresca and Mohr-Coulomb criteria. In particular, $\tau_{s f}$ is not the maximum possible shear strength. As will be seen later, the material parameter $f_{c}^{\prime}$ denotes the failure stress in uniaxial compression. Although the full quadratic form of the in-plane stress is selected for convenience, other forms can be chosen equally well if warranted by experimental data. For example, one possibility is to incorporate the in-plane stress only in compression:

$$
F=\max _{\forall \mathbf{n}} F_{n 1}\left(\boldsymbol{\tau}, \sigma_{t t}\right) \quad F_{n 1}=\frac{\tau_{t}^{2}}{\tau_{s f}^{2}}+\frac{\tau_{n}}{\tau_{n f}}+\frac{\left\langle-\sigma_{t t}\right\rangle^{2}}{f_{c}^{\prime 2}}-1
$$

where $\langle\mathrm{x}\rangle=\mathrm{x}$ if $\mathrm{x}$ is positive; otherwise $\langle\mathrm{x}\rangle$ is zero. The term involving the in-plane stress could be linear, or any other power if necessary to match experimental data. If it is believed that the strength in shear should reach a limiting value for large negative values of $\tau_{n}$, then an exponential form can be utilized to relate the "brittle" and shear contributions:

$$
F=\max _{\forall \mathbf{n}} F_{n 2}\left(\boldsymbol{\tau}, \sigma_{t t}\right) \quad F_{n 2}=\frac{\tau_{t}^{2}}{\tau_{s f}^{2}}+e^{\kappa\left(\frac{\tau_{n}}{\tau_{n f}}+\frac{\left\langle\left.\sigma_{t t}\right|^{2}\right.}{f_{c}^{\prime 2}}-1\right)}-1
$$

with $\kappa$ an additional material parameter. The form (8) has been used in numerical simulations by Sulsky et al. [2006]. Here, (6) is used for analytical convenience and simplicity in presenting the essential ideas.

[30] The classical surfaces and the proposed decohesion surface of (6) are displayed in the space of $\tau_{t}$ and $\tau_{n}$ in Figures $3 \mathrm{a}$ and $3 \mathrm{~b}$, respectively. Note that the shear contribution must be zero for the Rankine criterion because, by definition, the normal to the failure surface is a direction of maximum principal stress so any shear component associated with this surface is zero. As indicated in Figure $3 \mathrm{~b}$, the quadratic term for shear in (6) provides a smooth transition at $\tau_{t}=0$ whereas the Mohr-Coulomb criterion introduces a discontinuity in slope. When the normal component of traction is sufficiently compressive, the quadratic form indicates a failure stress in shear that is intermediate to those given by the Mohr-Coulomb and Tresca criteria. Also, when the in-plane stress becomes $\sigma_{t t}=-f_{c}^{\prime}$, failure is predicted even if $\tau_{n}$ and $\tau_{\mathrm{t}}$ are zero. Note that failure is also predicted for a tensile value of $\sigma_{t t}=f_{c}^{\prime}$. However, for realistic values of material parameters, the tensile failure stress of $\tau_{n f}$ for a different orientation of $\mathbf{n}$ would be activated and a tensile stress of $f_{c}^{\prime}$ can never be realized.

[31] If the shear parameter, $\tau_{s f}$, is infinite then (6) reduces to

$$
B=\max _{\forall \mathbf{n}} B_{n} \quad B_{n}=\frac{\tau_{n}}{\tau_{n f}}+\frac{\sigma_{t t}^{2}}{f_{c}^{\prime 2}}-1
$$

The surface $\mathrm{B}=0$ is one for which $\mathbf{n}$ is always in the direction of maximum principal stress and, consequently, represents a brittle criterion. With the use of (9), the decohesion function of (6) can be rewritten as the sum of shear and brittle parts as follows:

$$
F_{n}=\frac{\tau_{t}^{2}}{\tau_{s f}^{2}}+B_{n}
$$


Note that $B_{n} \leq 0$ because no solution to $F_{n}=0$ exists for $B_{n}>0$.

\subsection{Analytical Form of the Critical Direction}

[32] Performing a numerical search to find the maximum value of $F$ with respect to $\theta$ is computationally intensive and might result in unacceptable run times for large-scale simulations involving failure of Arctic ice. Because of the relatively simple form of (6) it is possible to find analytically the critical value of $\theta$ by setting $\partial F / \partial \theta=0$. As a first step, it is convenient to transform to the principal basis of stress with principal stresses ordered such that $\sigma_{1} \geq \sigma_{2}$. Then the transformation relations for the components in the local $\mathbf{n}$-t system reduce to

$$
\begin{aligned}
\tau_{n} & =\sigma_{1} \cos ^{2} \theta+\sigma_{2} \sin ^{2} \theta \\
\tau_{t} & =\left(\sigma_{2}-\sigma_{1}\right) \cos \theta \sin \theta \\
\sigma_{t t} & =\sigma_{1} \sin ^{2} \theta+\sigma_{2} \cos ^{2} \theta
\end{aligned}
$$

After the derivatives with respect to $\theta$ of the terms in (11) have been obtained, it follows that

$$
\frac{\partial F_{n}}{\partial \theta}=\left(\sigma_{1}-\sigma_{2}\right) \sin 2 \theta\left[\frac{\left(\sigma_{1}-\sigma_{2}\right)}{\tau_{s f}^{2}} \cos 2 \theta-\frac{1}{\tau_{n f}}+\frac{2 \sigma_{t t}}{f_{c}^{\prime 2}}\right]
$$

Then $\partial F_{n} / \partial \theta=0$ if $\theta=\theta_{c}$ where $\theta_{c}=0$ or $\theta_{c}=\pi / 2$. The latter is associated with a maximum of $F_{n}$ and will not be considered further. Another solution for $\theta_{c}$ is obtained by setting the square bracket to zero. The latter occurs if $\theta_{c}=\alpha$ and $\alpha$ is a solution to

$$
\tan ^{2} \alpha=\frac{\frac{\left(\sigma_{1}-\sigma_{2}\right)}{\tau_{s f}^{2}}-\frac{1}{\tau_{n f}}+\frac{2 \sigma_{2}}{f_{c}^{\prime 2}}}{\frac{\left(\sigma_{1}-\sigma_{2}\right)}{\tau_{s f}^{2}}+\frac{1}{\tau_{n f}}-\frac{2 \sigma_{1}}{f_{c}^{\prime 2}}}
$$

A solution to (13) does not exist if the right side is less than zero; otherwise there are actually two solutions $\theta_{c}= \pm \alpha$.

[33] As an example, consider uniaxial tension with $\sigma_{1}=\tau_{n f}$ and $\sigma_{2}=0$. Then a solution other than $\theta_{c}=0$ does not exist unless $\tau_{s f}<\tau_{n f}$.

[34] Similarly, for uniaxial compression with $\sigma_{1}=0$ and $\sigma_{2}=-f_{c}^{\prime}$, (13) yields

$$
\tan ^{2} \alpha=\frac{\frac{f_{c}^{\prime}}{\tau_{s f}^{2}}-\frac{1}{\tau_{n f}}-\frac{2}{f_{c}^{\prime}}}{\frac{f_{c}^{\prime}}{\tau_{s f}^{2}}+\frac{1}{\tau_{n f}}}
$$

The critical orientation remains $\theta_{c}=0$, which corresponds to axial splitting, unless

$$
\tau_{s f} \leq f_{c}^{\prime} \sqrt{\frac{\tau_{n f}}{f_{c}^{\prime}+2 \tau_{n f}}}
$$

in which case two solutions exist and failure with a shear component exists.

\subsection{Effect of Material Parameters on Failure Surface}

[35] The results of the previous subsection can be used as a guide for choosing material parameters if sufficient experimental data are available. First, suppose $\tau_{n f}=0.1$

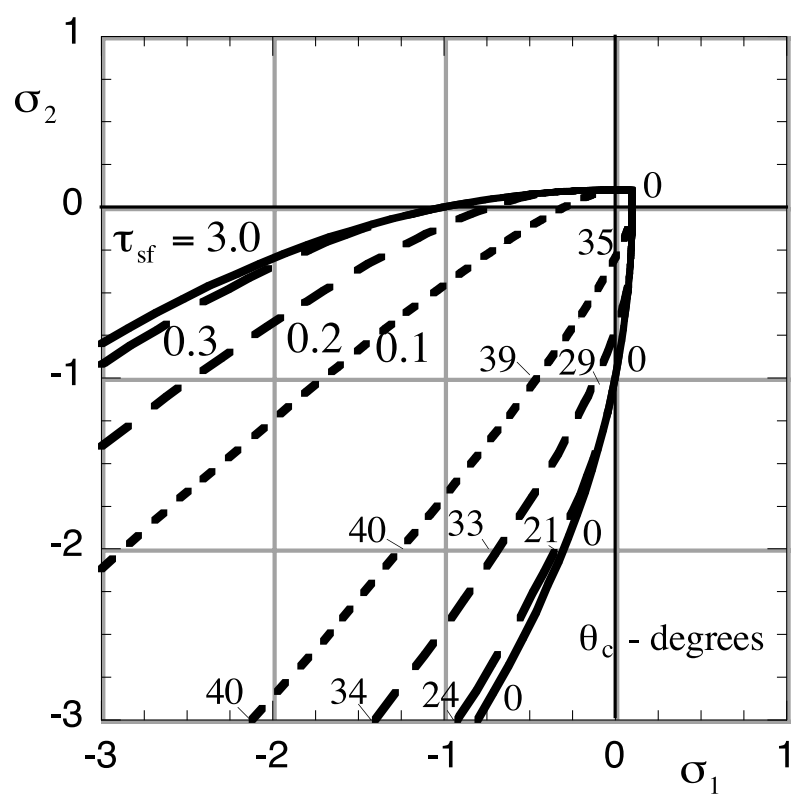

(a) Effect of $\tau_{\text {sf }}$ for $\tau_{\text {nf }}=0.1$ and $\mathrm{f}_{\mathrm{c}}^{\prime}=1.0$.

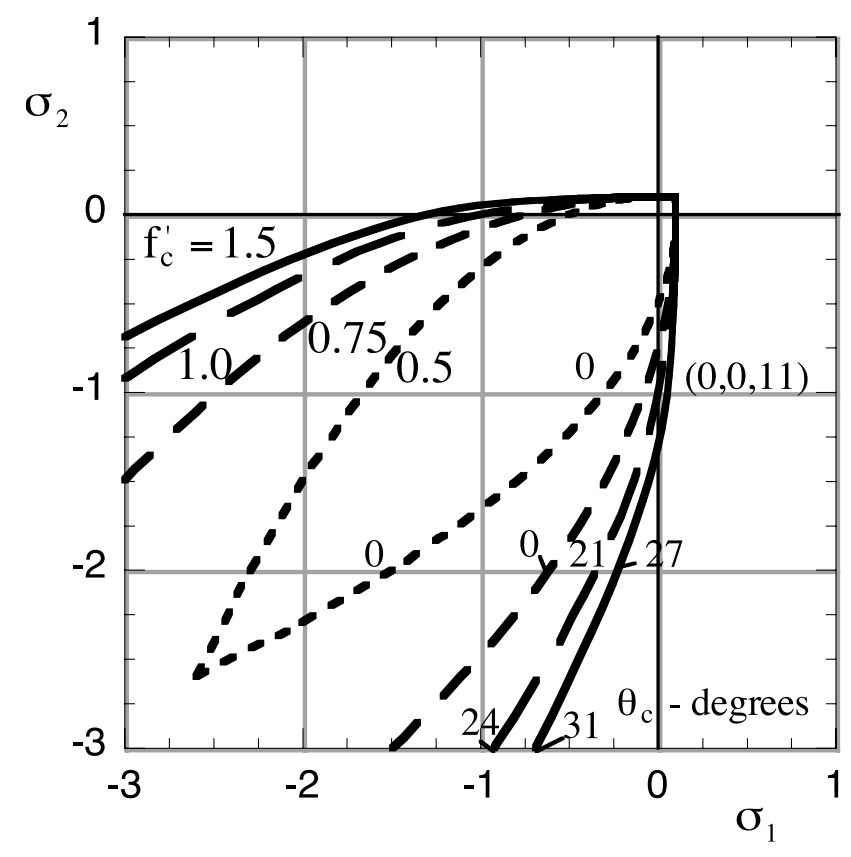

(b) Effect of $f_{c}^{\prime}$ for $\tau_{n f}=0.1$ and $\tau_{\text {sf }}=0.3$.

Figure 4. Effects of material parameters on shape of decohesion surface for plane stress.

and $\mathrm{f}_{\mathrm{c}}^{\prime}=1.0=10 \tau_{n f}$. Figure $4 \mathrm{a}$ shows a sequence of surfaces based on different values for $\tau_{s f}$ as specified on the left side of the plot. The orientation of the normal as defined by $\theta_{c}$ is given on the right side. Only positive values are stated even though negative signs for the values shown are also 
solutions. When $\tau_{s f}=3.0$, the outer surface is obtained and $\theta_{c}=0$ for all points on the surface, i.e., the normal is in the direction of maximum principal stress. When $\tau_{s f}=0.3$, a value close to the result obtained from the right side of (15), the next surface is obtained. For the regime from equal biaxial tension down to uniaxial compression $\theta_{c}=0$ so uniaxial compressive splitting is still predicted. However, when both components become compressive, $\theta_{c}$ quickly increases from zero. For the other two cases identified by $\tau_{s f}=0.2$ and $\tau_{s f}=0.1$ the normal is still $\theta_{c}=0$ when both principal stresses are tensile, but the transition to large values of $\theta_{c}$ is rather abrupt.

[36] The situation is somewhat different when $\tau_{n f}$ and $\tau_{s f}$ are fixed and $f_{c}^{\prime}$ is allowed to change as shown in Figure $4 \mathrm{~b}$. For these surfaces, $\tau_{n f}=0.1$ and $\tau_{s f}=0.3$. For the two innermost surfaces identified as $f_{c}^{\prime}=0.5$ and $f_{c}^{\prime}=$ 0.75 , the normal is in the direction of maximum principal stress $\left(\theta_{c}=0\right)$. The fact that these surfaces come together for large pressure is considered to be irrelevant for the current application because there is no evidence of failure in the icepack for a stress state in the regime of large equal biaxial compressive stresses. Evidence of such a failure would be a material failure surface with a normal predominately out of the plane and no photographs of failure within pack ice show such an orientation. Failure of thin fresh ice formed within a lead is another matter that is discussed in section 5. The last two surfaces associated with $f_{c}^{\prime}=1.0$ and $f_{c}^{\prime}=1.5$ in Figure $4 \mathrm{~b}$ show rather abrupt transitions to nonzero values of $\theta_{c}$ as the value of either principal stress decreases from the tensile regime.

\subsection{Selection of Values for Material Parameters}

[37] The strength model of (6) contains three material parameters $\tau_{\mathrm{nf}}, \tau_{\mathrm{sf}}$ and $f_{c}^{\prime}$. An example of values based on laboratory data is given by Zubov [1943], who suggests a tensile strength of $\tau_{n f} \approx 0.1 \mathrm{MPa}$, a compressive strength of approximately 10 times the tensile strength, and a shear strength of $1 / 2$ the tensile strength. A tensile strength of $\tau_{n f} \approx 0.2 \mathrm{MPa}$ is given by Bazant [2002].

[38] Although laboratory data provide quantitative values for material parameters, and indicate failure modes of ice, there is no evidence that such data can be used directly in large-scale simulations. Two reasons could be: (1) imperfections such as thermal bending cracks that exist on a scale of a kilometer, and (2) preexisting leads that have closed and have not attained the strength of the surrounding ice. If laboratory data cannot be used, what values of material parameters should be used?

[39] In an attempt to provide guidance, in situ measurements of stress have been provided by Coon et al. [1998, Figure 3] in the form of pressure and shear invariants of stress:

$$
P=\frac{1}{2}\left(\sigma_{1}+\sigma_{2}\right) \quad \mathrm{S}_{\mathrm{h}}=\frac{1}{2}\left|\sigma_{1}-\sigma_{2}\right|
$$

A reasonable failure criterion might be an upper bound or envelope to these data. Coon et al. [1998] have provided a Mohr-Coulomb envelope to these data and have made the reasonable suggestion that this bound might serve as a failure surface. Such an argument suggests that a lower bound to the resultant shear strength is approximately $50 \mathrm{kN} / \mathrm{m}$ when the pressure is zero. For a depth of ice estimated to be $2.5 \mathrm{~m}$, the lower bound to the shear strength becomes $20 \mathrm{kPa}$ or $0.02 \mathrm{MPa}$ which is an order of magnitude less than the values suggested by Zubov and Bazant. A further analysis of scale effects by Coon et al. [1999] indicates that in order to obtain agreement between predicted and observed motions for large scale simulations, other researchers had to use even smaller values for strength.

[40] One other observation is critical for our objective of suggesting reasonable values for material parameters for pack ice. Based on in situ data from oil pressure sensors in a buoy, the data of Figure 7 in Coon et al. [1998] suggest that large-scale axial splitting may occur. For this case, a compressive failure stress of approximately $f_{c}^{\prime}=100 \mathrm{kPa}$ is reported. As one might expect, after failure, the in-plane component $\sigma_{t t}$ did not go to zero but remained at a significant value of around $60 \mathrm{kPa}$ in compression.

[41] For the proposed model, suppose the compressive and tensile strengths are chosen to be $\tau_{n f}=20 \mathrm{kPa}$ and $f_{c}^{\prime}=$ $100 \mathrm{kPa}$. The assumption that axial splitting may occur requires that $\tau_{s f}$ meets the condition of (15) and a value of $\tau_{s f}=38 \mathrm{kPa}$ satisfies this inequality. Plots of the resulting surface in principal stress space and in pressure-shear space are given in Figures $5 \mathrm{a}$ and $5 \mathrm{~b}$, respectively.

[42] Also shown in Figure 5 are the corresponding representations of the Mohr-Coulomb surface obtained by using (5). The values for the material parameters $\left(\tau_{n f}^{M C}=\right.$ $24 \mathrm{kPa}$ and $\tau_{s f}^{M C}=23 \mathrm{kPa}$ ) were chosen to provide the surface in Figure 5b that replicates the surface provided by Coon et al. [1999] with an adjustment from stress resultant to stress. Although the shapes of the Mohr-Coulomb surface and the proposed surface are similar in the region of most interest for pack ice, Figure 5a shows that there is a large difference in the prediction of the normal to the material failure surface. To emphasize this point even further, if the signs of both principal stresses are positive, then the MohrCoulomb model predicts a normal with a component out of the plane whereas the normal to the failure surface for the proposed model lies in the direction of maximum principal stress $\left(\theta_{c}=0\right)$.

[43] The proposed model can be viewed as a generalization of classical models with an important distinction. The use of the in-plane normal stress in the proposed formulation results in a prediction of the orientation of the surface of material failure that can vary with the state of stress. Such a variation is in accord with experimental evidence based on specimens of both fresh and salt ice and is also true of experimental data for other geological materials [Lade, 2001].

[44] For pack ice, when both principal stresses are large and negative, failure is generally not observed so the model makes no attempt to provide a closed surface for the regime close to equal biaxial stress in compression. If such stresses were developed, the failure would probably reflect a component that is out of the plane, a feature purposely excluded in the present model based on simplicity and on potential application to pack ice. However, the model does provide axial splitting under uniaxial compression a feature that is 


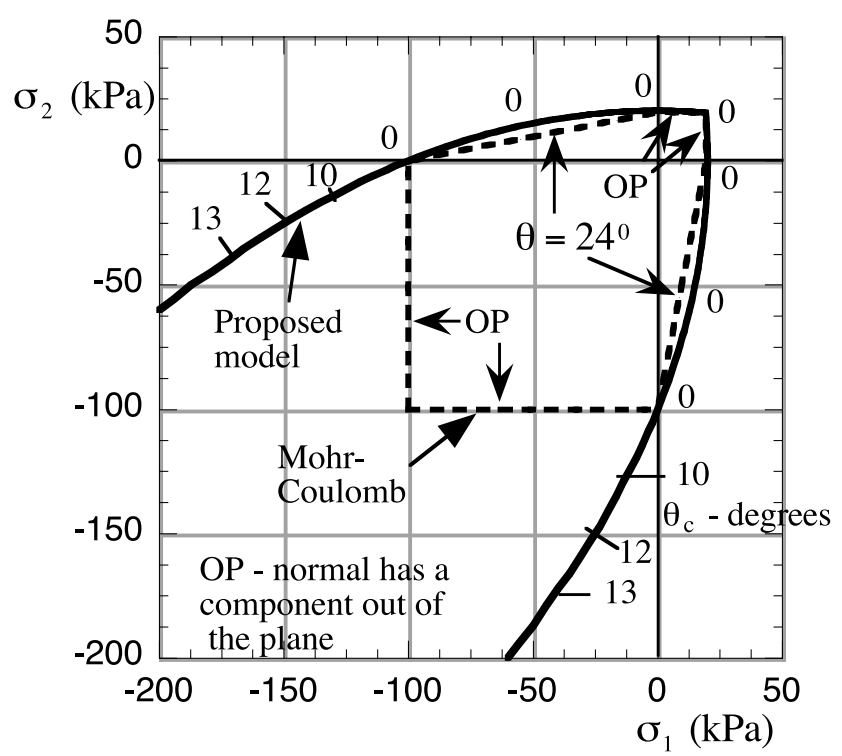

(a) Surfaces in principal-stress space.

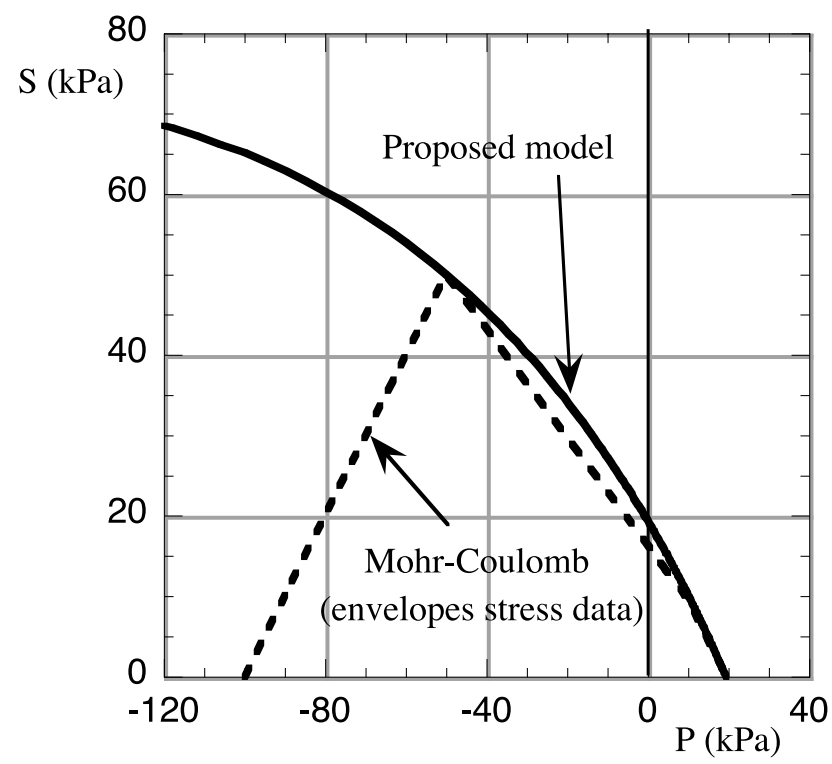

(b) Surfaces in P-S space.

Figure 5. Failure surfaces based on an envelope of ice stress measured in situ by Coon et al. [1998].

observed on both small and large scales. A reduction by an order of magnitude of values of material parameters obtained from laboratory specimens must be performed to reflect the large-scale imperfections of thermal cracks and frozen leads exhibited by pack ice.

[45] After determining the initiation of failure and the orientation of the material surface of failure, the next issue is that of determining the mode of failure and of providing a method for describing the evolution of failure, the topic of the following section.

\section{Evolution of Failure}

\subsection{Displacement Discontinuity and Softening}

[46] The basic assumption inherent with a decohesive approach to modeling failure is that a discontinuity in displacement, $[\mathbf{u}]$, develops simultaneously with a reduction in traction. Decohesion is defined to be the loss of strength that occurs with an increase in a suitable norm of the displacement discontinuity. Such an approach is inherently different from classical fracture mechanics for which a stress singularity exists and the issue of whether or not a crack propagates is associated with a critical stress intensity factor. A decohesive or discrete framework implies that propagation is identified with failure initiation provided by a critical value of a decohesion function.

[47] For the planar problem considered here, it is convenient to express the displacement discontinuity in terms of normal and tangential components

$$
[\mathbf{u}]=\left[u_{n}\right] \mathbf{n}+\left[u_{t}\right] \mathbf{t}
$$

In analogy with some models of plasticity, it is assumed that a single scalar parameter, $\bar{u}$, called the effective decohesion, can be used to quantify the degree of decohesion that has occurred. The parameter, $\bar{u}$, is monotonically increasing and obtained as an integral of its time derivative which is defined in terms of the rate of the displacement discontinuity as follows

$$
\dot{\bar{u}}=\{[\dot{\mathbf{u}}] \cdot \mathbf{D} \cdot[\dot{\mathbf{u}}]\}^{1 / 2}
$$

The positive definite, second-order tensor, $\mathbf{D}$, depends on the material and is defined to yield the correct ratio of fracture energy for Modes I (pure opening) and II (pure shear).

[48] Under uniaxial tension, it is assumed that decohesion does not begin until the traction $\tau_{n}$ attains the critical value $\tau_{n f}$. Then $\tau_{n}$ decreases as $\left[u_{n}\right]$ increases until a critical value $\left[u_{n}\right]=u_{0}$ is reached at which all tractioncarrying capability is lost and two free surfaces have been created. For ice, it is assumed that a purely shear mode of failure does not exist or, in other words, $\left[u_{n}\right]$ always increases during failure. Then, for simplicity, $\mathbf{D}$ is chosen so that, (18) yields an effective decohesion that involves only the normal component

$$
\dot{\bar{u}}=\left[\dot{u}_{n}\right] / u_{0}
$$

In this work, issues of closing when a crack has only partially formed will not be considered so that $\left[u_{n}\right]$ is, itself, a monotonically increasing parameter.

[49] Again, for simplicity a linear decay is assumed so that $\tau_{n}=\tau_{n f} f_{n}$ in which $f_{n}$ is a softening function associated with the direction $\mathbf{n}$ as follows:

$$
\begin{array}{ll}
f_{n}=1-\bar{u} & \text { if } \quad \bar{u} \leq 1 \\
f_{n}=0 & \text { if } \quad \bar{u}>1
\end{array}
$$


The result is that for uniaxial tension, the normal traction is related linearly to the normal component of displacement discontinuity as follows:

$$
\tau_{n}=\tau_{n f}\left[1-\frac{\left[u_{n}\right]}{u_{0}}\right]
$$

[50] For the sake of simplicity, if the internal energy associated with the material failure surface is ignored, then the fracture energy, $g_{f}$, is obtained from the relation

$$
g_{f}=\int_{0}^{u_{0}} \tau_{n}\left[d u_{n}\right]=\frac{1}{2} \tau_{n f} u_{0}
$$

The parameter $u_{0}$ can be determined if $\tau_{n f}$ and $g_{f}$ are given as material constants. Schreyer et al. [2002] provide a more general formulation.

[51] In order to consider any state of stress, not just one of uniaxial tension, the softening function is included in the decohesion function in such a manner that if $\tau_{n}$ is positive, then both components of traction $\tau_{n}$ and $\tau_{s}$ should approach zero as $f_{n}$ goes to zero. Such a property is obtained if the decohesion function of (6) is changed to the following:

$$
F=\max _{\forall \mathbf{n}} F_{n} \quad F_{n}=\frac{\tau_{t}^{2}}{\tau_{s f}^{2}}+\frac{\tau_{n}}{\tau_{n f}}+f_{n}\left(\frac{\sigma_{t t}^{2}}{f_{c}^{\prime 2}}-1\right)
$$

The effect of $f_{n}$ on the decohesion surface, $F=0$, in the $\tau_{n}-\tau_{t}$ space is shown in Figure 6 for $\left(\sigma_{t t}^{2} / f_{c}^{\prime 2}\right)=0.5$. In particular, when failure is complete, then $f_{n}=0$, and shear can still be sustained when $\tau_{n}$ is negative. Although the increase in shear with negative $\tau_{n}$ appears to be unbounded; in reality, another failure surface will be activated when the stress gets large enough.

\subsection{Evolution Equations}

[52] The mode of failure, $\mathbf{m}$, is defined to be the direction of the discontinuity in displacement:

$$
\mathbf{m}=[\mathbf{u}] /|[\mathbf{u}]|
$$

An associated evolution equation is proposed with $\omega$ defined to be a monotonically increasing parameter that is a measure of the amount of decohesion. With a scale factor $\mathrm{S}_{\mathrm{F}}$ chosen to render $\omega$ dimensionless, an associated rule is

$$
\left[\dot{u}_{n}\right]=\dot{\omega} S_{F} \frac{\partial F_{n}}{\partial \tau_{n}} \quad\left[\dot{u}_{t}\right]=\dot{\omega} S_{F} \frac{\partial F_{n}}{\partial \tau_{t}}
$$

in which superposed dots denote derivatives with respect to time. Because the time derivative appears linearly on both the left and right sides of each equation, any variable that increases monotonically with time can also be used as a time variable. Expressed another way, the formulation is rate independent. Suppose the scale factor is chosen to be

$$
S_{F}=u_{0} \tau_{n f}
$$

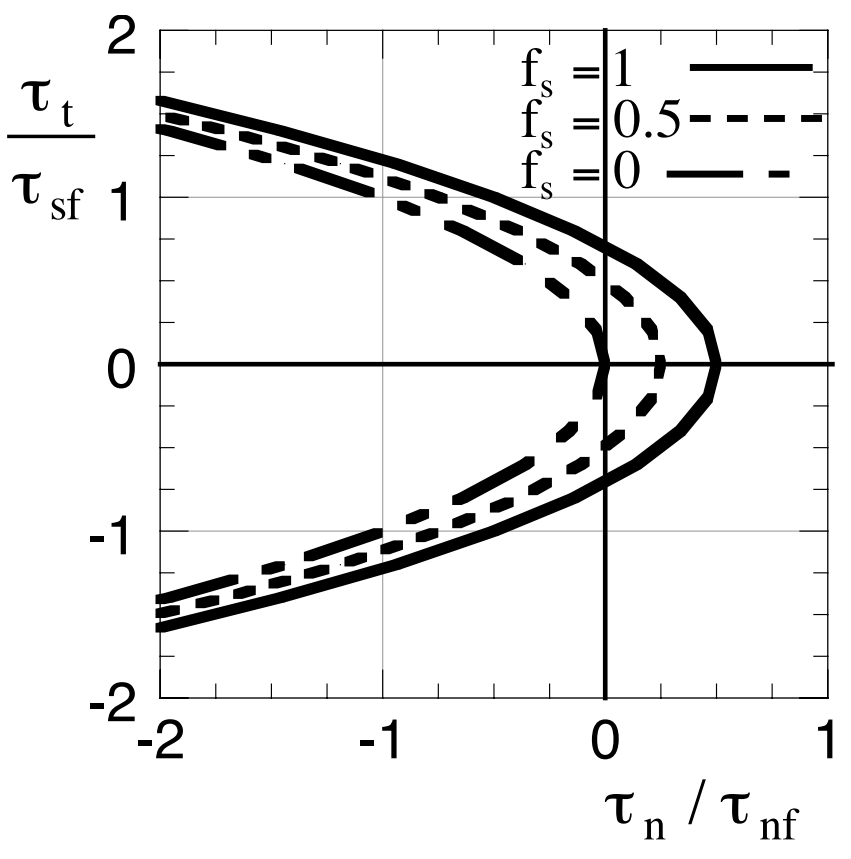

Figure 6. Effect of the value of the softening function on the decohesion surface in the $\tau_{n}-\tau_{t}$ space for $\left(\sigma_{\mathrm{tt}} / \mathrm{f}_{\mathrm{c}}^{\prime}\right)^{2}=0.5$.

Then (25) becomes

$$
\left[\dot{u}_{n}\right]=u_{0} \dot{\omega} \quad\left[\dot{u}_{t}\right]=2 u_{0} \dot{\omega} \frac{\tau_{t}}{\tau_{s f}} \frac{\tau_{n f}}{\tau_{s f}}
$$

[53] The use of (19) implies that

$$
\dot{\omega}=\dot{\bar{u}}
$$

so that the decohesion parameter, $\omega$, is dimensionless, as desired, and has the physical interpretation associated with $\bar{u}$.

[54] An important attribute of the formulation is that the normal component of displacement discontinuity continues to increase for all states of stress satisfying the decohesion condition, in particular for uniaxial compression is which case the normal component of traction, $\tau_{n}$, is zero. This is considered to be a desirable and unique feature of the model and is a consequence of incorporating $\tau_{n}$ as a linear function in the expression for the decohesion function given by (23).

[55] In addition to (27), one equation is necessary for determining $\dot{\omega}$. The equation necessary for closure is that of consistency which states that the decohesion condition $F_{n}=0$ must continue to be satisfied or, expressed in rate form,

$$
\dot{F}_{n}=0
$$

[56] At this time, it is noted that if the normal to the failure surface is in the direction of maximum principal stress, then $\tau_{t}=0$ and (27) implies that the mode of deformation is in the direction of the normal, a situation that is typical for brittle failure. This is the reason why the decohesion function of (9) is characterized as a brittle one. 


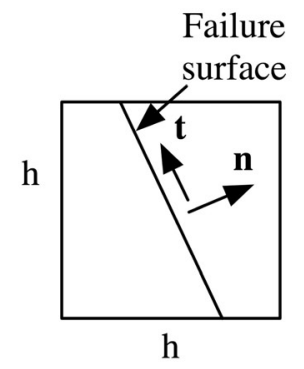

Original square element with failure initiation

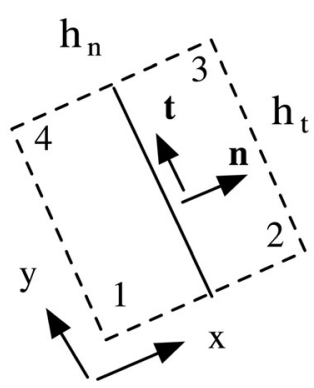

Oriented

"equivalent" element

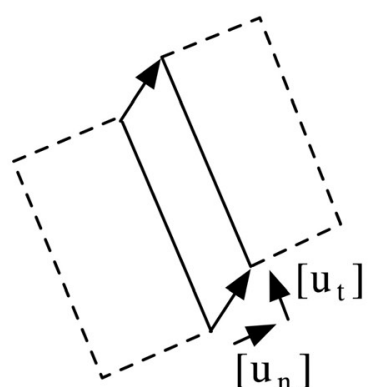

Deformation
due to
decohesion

Figure 7. Relationship between original square element and equivalent element used to define decohesive strains.

[57] Once a complete crack, as reflected by $f_{n}=0$, has been established the evolution equations of (27) can no longer be applied. Instead, alternative equations must be invoked. Nonzero traction is physically unacceptable if the free surfaces of a lead have separated so the appropriate condition then is to adjust the components of displacement discontinuity (or decohesive strains as will be shown later) so that $\tau_{n}=0$ and $\tau_{t}=0$. If the lead is forced closed so that $\tau_{n}<0$ then the value of the modified decohesion function

$$
\left.\mathrm{F}_{\mathrm{n}}^{*} \equiv F_{n}\right|_{f_{n}=0}=\frac{\tau_{t}^{2}}{\tau_{s f}^{2}}+\frac{\tau_{n}}{\tau_{n f}}
$$

must be checked. If $F_{n}^{*} \leq 0$ no further action is required. However, if $\mathrm{F}_{\mathrm{n}}^{*}>0$ then the shear decohesion strain should be increased or decreased in order to adjust the magnitude of $\tau_{t}$ and force $\mathrm{F}_{\mathrm{n}}^{*}$ back to zero.

\section{Elastic-Decohesion Model}

\subsection{Combined Elasticity and Decohesion}

[58] Any continuum constitutive equation that is considered appropriate prior to failure can be used to describe the pack ice. For the sake of simplicity it is assumed that linear, isotropic elasticity is adequate. For plane stress in the $x_{1}-x_{2}$ plane, the relations between stress and strain rate in component form are

$$
\begin{aligned}
& \dot{\sigma}_{11}=E_{1} \dot{e}_{11}+E_{2} \dot{e}_{22} \\
& \dot{\sigma}_{22}=E_{2} \dot{e}_{11}+E_{1} \dot{e}_{22} \\
& \dot{\sigma}_{12}=2 G \dot{e}_{12}
\end{aligned}
$$

in which the elasticity parameters are

$$
E_{1}=Y \frac{(1-\nu)}{(1+\nu)} \quad E_{2}=Y \frac{\nu}{\left(1-\nu^{2}\right)} \quad 2 G=Y \frac{1}{(1+\nu)}
$$

and $\mathrm{Y}$ denotes Young's modulus, $\nu$ Poisson's ratio and $\mathrm{G}$ the shear modulus.
[59] For a material point at which failure is occurring, the object is to solve (31) subject to the constraint that the consistency condition and the evolution equations of (27) are satisfied. This combination of equations is what is meant by the phrase "elastic-decohesion constitutive equations".

\subsection{Numerical Treatment of Elastic Decohesion Constitutive Equations}

[60] Although the constitutive equations have been given, there remains the question of how these equations are combined and implemented with an algorithm for solving the equations of motion and of deformation. Discontinuities in displacement represent a relatively new area of analysis and a general consensus on the most appropriate method has not been achieved.

[61] Even for the finite element method, which is widely used in the engineering community, there are several methods for kinematically representing jumps in displacement. In what is called the extended finite element method, one approach is to use enhanced basis functions so that the discontinuity across element boundaries is modeled explicitly of which representative examples are given by Moës et al. [1999], Wells and Sluys, [2001a, 2001b, 2001c], and Alfaiate et al. [2003]. An alternative procedure is to introduce a localized band within an element [Larsson and Runesson, 1996]. A more elementary method is to assume that a crack displacement field is constant over an element and to smear the effect over the element [Rashid, 1968] an approach that is almost as old as the finite element method itself. The smeared crack approach has been subject to considerable criticism but it is believed that some of the noted shortcomings should actually be attributed to limitations of the constitutive equations and not the method itself. Because the smeared crack method has many positive attributes, not least of which is its simplicity, the procedure described next is based on the method. For the limited range of applications considered here, the method is considered to be adequate.

[62] Consider an active crack with current components of discontinuity $\left[u_{n}\right]$ and $\left[u_{t}\right]$, assumed to be constant across a square element of side $h$ as indicated in Figure 7. For a 
crack with a normal $\mathbf{n}$ oriented at an angle $\theta$ to the $\mathrm{x}_{1}$-axis, the length of the crack is denoted by $h_{t}$ where

$$
\begin{array}{ll}
h_{t}=\frac{h}{\cos \theta} & -\frac{\pi}{4} \leq \theta \leq \frac{\pi}{4} \\
h_{t}=\frac{h}{\sin \theta} & \frac{\pi}{4} \leq \theta \leq \frac{3 \pi}{4}
\end{array}
$$

A general expression for the rate of decohesive strain tensor within a representative strip of material of width $L^{d c}$ containing the crack is

$$
\dot{\mathbf{e}}^{d c}=\frac{1}{L^{d c}}\{[\dot{\mathbf{u}}] \otimes \mathbf{n}\}_{s y m} \quad \dot{e}_{i j}^{d c}=\frac{1}{2 L^{d c}}\left\{[\dot{u}]_{i} n_{j}+[\dot{u}]_{j} n_{i}\right\}
$$

The expression on the left is in direct notation and involves the tensor product, $\otimes$, which is defined such that the corresponding expression in indicial notation is shown on the right. For an element, the parameter, $L^{d c}$, is determined by equating the rate of dissipation considering the effect of the discontinuity as smeared over the element to the rate of dissipation performed by the traction acting on the surface discontinuity. If $\Omega$ denotes the area of the element, $\mathrm{dA}$ a differential of area, and dr a differential of crack length, equating dissipation rates yields

$$
\int_{\Omega} \boldsymbol{\sigma}: \frac{1}{L^{d c}}\{[\mathbf{u}] \otimes \mathbf{n}\}_{\text {sym }} d A=\int_{\mathrm{h}_{\mathrm{t}}} \boldsymbol{\tau} \cdot[\mathbf{u}] d r
$$

Now, assume the discontinuity is constant along the crack within an element, and the decohesive crack strain and stress are constant over an element. The result is

$$
L^{d c} \equiv h_{n}=\frac{h^{2}}{h_{t}}
$$

and (34) yields specific expressions for components of decohesive strain in the n-t basis as follows:

$$
e_{n n}^{d c}=\frac{\left[u_{n}\right]}{h_{n}} \quad e_{n t}^{d c}=\frac{\left[u_{t}\right]}{2 h_{n}} \quad e_{t t}^{d c}=0
$$

An alternative way of viewing the development is to visualize a rotated rectangular element (dotted lines in Figure 7) with sides of length $h_{n}$ and $h_{t}$ and an area equal to the original element. For this dotted element with the deformation due only to a uniform discontinuity in displacement, the displacement components at the nodes for the local coordinate system are

$$
\begin{aligned}
& u_{x}^{1}=0 \quad u_{x}^{2}=\left[u_{n}\right] \quad u_{x}^{3}=\left[u_{n}\right] \quad u_{x}^{4}=0 \\
& u_{y}^{1}=0 \quad u_{y}^{2}=\left[u_{t}\right] \quad u_{y}^{3}=\left[u_{t}\right] \quad u_{y}^{4}=0
\end{aligned}
$$

in which superscripts denote the node number and the subscript indicates the component. The basis functions for a 4-noded, rectangular element are

$$
\begin{aligned}
N_{1} & =\left(1-\frac{x}{h_{n}}\right)\left(1-\frac{y}{h_{t}}\right) & N_{2} & =\frac{x}{h_{n}}\left(1-\frac{y}{h_{t}}\right) \\
N_{3} & =\frac{x}{h_{n}} \frac{y}{h_{t}} & N_{4} & =\left(1-\frac{x}{h_{n}}\right) \frac{y}{h_{t}}
\end{aligned}
$$

If the displacement field is smeared over the element in the conventional continuous form, then

$$
\begin{aligned}
& u_{x}=u_{x}^{1} N_{1}+u_{x}^{2} N_{2}+u_{x}^{3} N_{3}+u_{x}^{4} N_{4} \\
& u_{y}=u_{y}^{1} N_{1}+u_{y}^{2} N_{2}+u_{y}^{3} N_{3}+u_{y}^{4} N_{4}
\end{aligned}
$$

The linear strain-displacement relations are

$$
e_{x x}=\frac{\partial u_{x}}{\partial x} \quad e_{y y}=\frac{\partial u_{y}}{\partial y} \quad e_{x y}=\frac{1}{2}\left(\frac{\partial u_{y}}{\partial x}+\frac{\partial u_{x}}{\partial y}\right)
$$

If $\mathrm{x}$ and $\mathrm{y}$ are associated with the unit vectors $\mathbf{n}$ and $\mathbf{t}$, respectively, and the strain components are evaluated at the center of the element after (38), (39) and (40) are substituted in (41), the result is identical to the components of the decohesive strain tensor given in (37).

[63] If there is no decohesion, the stress rates will simply be those given by (31). If there is decohesion, the stress rate must be relaxed in a manner such that traction continuity is maintained across the crack within the element. The result is an effective stress rate defined as follows:

$$
\begin{aligned}
& \dot{\sigma}_{11}=E_{1}\left(\dot{e}_{11}-\dot{e}_{11}^{d c}\right)+E_{2}\left(\dot{e}_{22}-\dot{e}_{22}^{d c}\right) \\
& \dot{\sigma}_{22}=E_{2}\left(\dot{e}_{11}-\dot{e}_{11}^{d c}\right)+E_{1}\left(\dot{e}_{22}-\dot{e}_{22}^{d c}\right) \\
& \dot{\sigma}_{12}=2 G\left(\dot{e}_{12}-\dot{e}_{12}^{d c}\right)
\end{aligned}
$$

in which the components of decohesive strain are obtained through the transformation relations from the component forms given in (37). Given the strain rates, $\dot{e}_{11}, \dot{e}_{22}$ and $\dot{e}_{12}$, it is the role of a constitutive equation subroutine to provide the stress rates, and the decohesive rates $\left[\dot{u}_{n}\right],\left[\dot{u}_{t}\right]$ and $\dot{\omega}$ such that (27) and (42) are satisfied simultaneously. The procedure is completely analogous to that used with computational plasticity.

[64] With computational algorithms, time steps and, hence, strain increments are finite and it is generally not feasible to maintain consistency for all time. Instead it is customary to assume that a step in prescribed strain is elastic and obtain a "trial" stress. The decohesion function is evaluated with the new values of stress (traction). If the value of the decohesion function is negative or zero, the step was truly elastic and no further modifications are necessary. If, on the other hand, $F_{n}>0$ then it is assumed to be satisfactory if displacement discontinuity and decohesive strains are increased according to the evolution equations until the value of the decohesion function is forced to zero only at the end of the step. One method for zero finding is the NewtonRaphson procedure which states that increments in the decohesion parameter are given by

$$
\Delta \omega=-\frac{F_{n}}{\partial F_{n} / \partial \omega}
$$

It has been assumed that $\omega$ is a monotonically increasing parameter, and since $F_{n}$ is being forced to zero from above, it follows that $\partial F_{n} / \partial \omega$ must be negative, a 
condition called a "stability" criterion. To evaluate $\partial F_{n} / \partial \omega$, the equation

$$
\dot{F}_{n}=\dot{\omega}\left(\partial F_{n} / \partial \omega\right)
$$

is used. First, note that as a consequence of isotropy, the elastic constitutive equations in the $\mathbf{n}$ - $\mathbf{t}$ system are of the same form as (42), or

$$
\begin{aligned}
& \dot{\tau}_{n}=E_{1}\left(\dot{e}_{n n}-\dot{e}_{n n}^{d c}\right)+E_{2}\left(\dot{e}_{t t}-\dot{e}_{t t}^{d c}\right) \\
& \dot{\sigma}_{t t}=E_{2}\left(\dot{e}_{n n}-\dot{e}_{n n}^{d c}\right)+E_{1}\left(\dot{e}_{t t}-\dot{e}_{t t}^{d c}\right) \\
& \dot{\tau}_{t}=2 G\left(\dot{e}_{n t}-\dot{e}_{n t}^{d c}\right)
\end{aligned}
$$

For this part of the algorithm, it is assumed that the total strains are fixed with the result that $\dot{e}_{n n}, \dot{e}_{t t}$ and $\dot{e}_{n t}$ are zero. Furthermore, $e_{t t}^{d c}$ is identically zero so (45) reduces to

$$
\dot{\tau}_{n}=-E_{1} \dot{e}_{n n}^{d c} \quad \dot{\sigma}_{t t}=-E_{2} \dot{e}_{n n}^{d c} \quad \dot{\tau}_{t}=-2 G \dot{e}_{n t}^{d c}
$$

The evolution equations of (27) and the decohesive strain relations of (37) yield

$$
\begin{aligned}
\dot{e}_{n n}^{d c} & =\frac{\left[\dot{u}_{n}\right]}{h_{n}}=\dot{\omega} \frac{u_{0}}{h_{n}} \\
\dot{e}_{n t}^{d c} & =\frac{\left[\dot{u}_{t}\right]}{2 h_{n}}=\dot{\omega} \frac{u_{0}}{h_{n}} \frac{\tau_{t}}{\tau_{s f}} \frac{\tau_{n f}}{\tau_{s f}}
\end{aligned}
$$

The use of (20) and (28) yields $\dot{f}_{n}=-\dot{\omega}$. It follows from (6), (46) and (47) that

$$
\begin{gathered}
\frac{\partial F_{n}}{\partial \omega}=-A \\
A=\frac{u_{0}}{h_{n}}\left[4 \frac{G}{\tau_{s f}} \frac{\tau_{t}^{2}}{\tau_{s f}^{2}} \frac{\tau_{n f}}{\tau_{s f}}+\frac{E_{1}}{\tau_{n f}}+f_{n} \frac{2 \sigma_{t t}}{f_{c}^{\prime}} \frac{E_{2}}{f_{c}^{\prime}}\right]-\left(1-\frac{\sigma_{t t}^{2}}{f_{c}^{\prime 2}}\right)
\end{gathered}
$$

The stability criterion is $\mathrm{A}>0$.

[65] To consider the implications of the stability criterion, first consider uniaxial tension so that $\tau_{t}=0$ and $\sigma_{t t}=0$. Then stability is satisfied provided

$$
\frac{u_{0}}{h_{n}} \frac{E_{1}}{\tau_{n f}}>1
$$

with the implication that the element size $h_{n}$ must be less than a critical value.

[66] For the case of $\tau_{t}=0$ and $\sigma_{t t} \neq 0, \mathrm{~A}$ is a minimum if

$$
\sigma_{t t}=-f_{n} \frac{u_{0}}{h_{n}} \frac{E_{1}}{\tau_{n f}} \frac{E_{2}}{E_{1}} \tau_{n f}
$$

and

$$
A_{\min }=\frac{u_{0}}{h_{n}} \frac{E_{1}}{\tau_{n f}}-1-f_{n}^{2}\left(\frac{u_{0}}{h_{n}} \frac{E_{1}}{\tau_{n f}}\right)^{2}\left(\frac{\tau_{n f}}{f_{c}^{\prime}}\right)^{2}\left(\frac{E_{2}}{E_{1}}\right)^{2}
$$

The last term does have an adverse effect on stability but since $\left(E_{2} / E_{1}\right)<1$ and, typically, $\left(\tau_{n f} / f_{c}^{\prime}\right) \simeq 0.1$ the effect on the critical value for $h_{n}$ will be small.

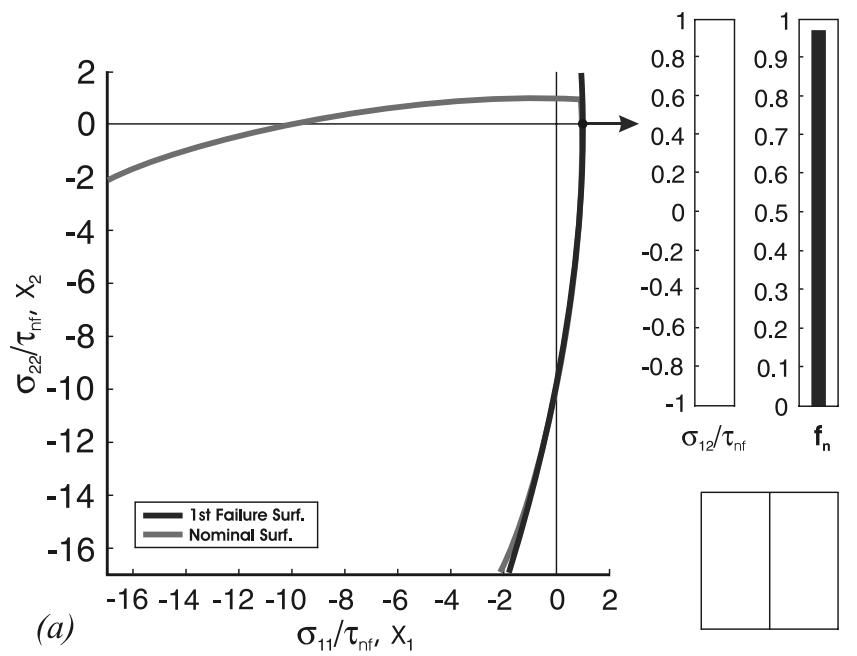

Figure 8a. Failure surfaces at the beginning of failure $\left(f_{n}=0.97\right)$ for uniaxial tensile stress.

[67] Because the first term in the square bracket of (48) is positive semi-definite, the addition of shear can only increase the critical value for $h_{n}$.

\subsection{Behavior With One Failure Surface}

[68] In this subsection representative paths in stress space are chosen to illustrate further the features of the model and to show implications of the evolution equations. Although the initial shape of the decohesion surface is isotropic in stress space, once a microcrack initiates, the decohesion surface displays anisotropy.

[69] The choice of the following material parameters is based on the assumption that $f_{c}^{\prime}$ is known. A Young's modulus of $Y=10^{3} f_{c}^{\prime}$, a Poisson's ratio of $\nu=0.36$ and a tensile strength of $\tau_{n f}=0.1 f_{c}^{\prime}$ are representative of a number of geological materials. As indicated in Figure 4a, a value for the shear-strength parameter of $\tau_{s f}=3 \tau_{n f}$ provides a transition point from brittle to ductile effects just slightly below and to the left of the uniaxial compression point, $\left(\sigma_{11}=0, \sigma_{22}=-f_{c}^{\prime}\right)$, within the compressive regime so that axial splitting can be predicted. The result is an initial decohesion surface (grey line) that is isotropic as shown in Figure $8 \mathrm{a}$ for $f_{c}^{\prime}=1$. Also shown are two bar charts, one to show shear stress and the other to plot one or two softening functions. Even if restrictions are made to consider paths only in principal strain space, shear stress may develop once a microcrack initiates. Instead of plotting stress in three dimensions, the choice has been made to use the first bar chart to show explicitly the development of a scaled value of shear stress. For certain paths, a shear stress will not develop. For simplicity, a restriction has been to limit the maximum number of cracks at any one point to two so the remaining bar chart is used to monitor the softening functions associated with microcracks. An initial value for each softening function is always unity. As a microcrack evolves, the value of its associated softening function will decrease. Finally, in the lower right corner is a coordinate axis on which is superimposed a schematic representation of a crack once it initiates.

[70] The first path of consideration is that of uniaxial tensile stress obtained in the elastic regime by specifying monoton- 

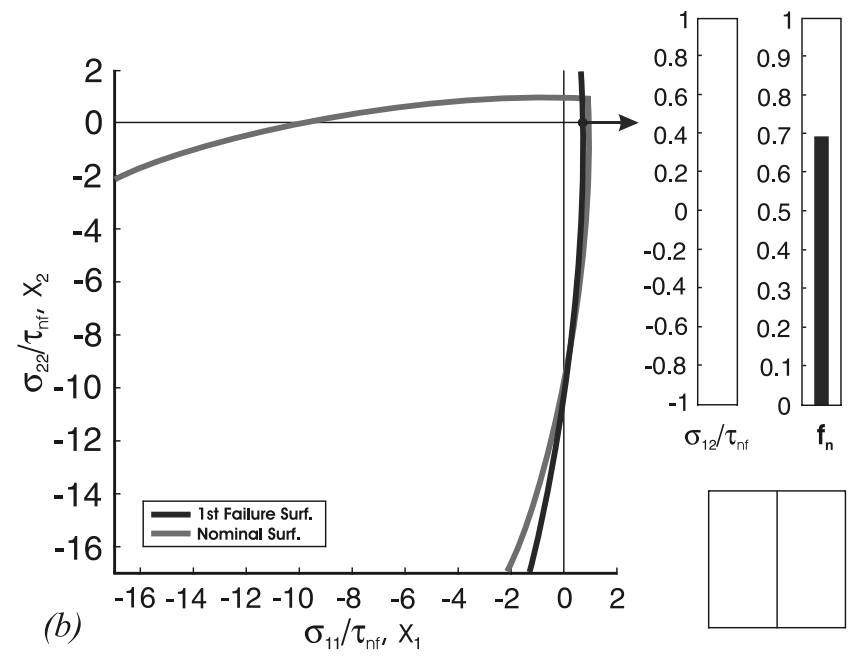

Figure 8b. Failure surfaces for uniaxial tensile stress when $\mathrm{f}_{\mathrm{n}}=0.68$.

ically increasing values for the strain $e_{11}$ with $e_{22}=-\nu e_{11}$. Once microcracking initiates, the component of strain $e_{11}$ continues to increase but the lateral strain is adjusted to enforce uniaxial stress. In this manner, a strain-driven algorithm can always be used whether the path is prescribed in strain or stress space. Figure 8a shows the initial decohesion surface, a shear stress of zero and the start of failure since the stress has just reached the initial decohesive surface and $f_{n}$ is slightly below one for the first surface.

[71] As loading continues, a microcrack is initiated with normal in the $\mathrm{x}_{1}$-direction as indicated by the appearance of an arrow in the direction of the normal to the failure surface located at the point of the current value of stress (Figures 8a and $8 \mathrm{~b}$ ). The original isotropic decohesive failure surface (grey line) is always included to provide a reference that helps to indicate the amount of change in the failure surface and to emphasize that the current surface is anisotropic. To show the orientation of the microcrack, a vertical line (the microcrack) is drawn in the lower right corner and a value of $f_{n}$ slightly less than one in the bar chart indicates the microcrack has just been initiated. Also shown in the stress space is the segment of the decohesive surface for $\theta=0^{\circ}$ with $f_{n}<1$. When decohesion is initiated, the current decohesive surface for a material point consists of a combination of segments of decohesive surfaces closest to the origin in stress space. For example, once decohesion occurs for $\theta=0^{\circ}$ with $f_{n}<1$, the top portion of the decohesive surface consists of the grey line; then the dark line through the point of uniaxial compression $\left(\sigma_{11} / \tau_{n f}=0, \sigma_{22} / \tau_{n f}=-10\right)$ to the transition point; and then the grey line again for stress points to the left and below the transition point $\sigma_{22} / \tau_{n f}<-10$. Therefore, Figure $8 \mathrm{~b}$ shows the appearance of anisotropy once a microcrack appears.

[72] Figures 8c and 8d show the continued development of decohesion as the value of the softening function decreases and the stress component $\sigma_{11}$ decreases to zero at which time failure is complete and part of the decohesion surface lies along the $\sigma_{22}$ axis. Note that the tensile and compressive strengths of the material for $\sigma_{22}$ and the compressive strength for $\sigma_{11}$ are unaffected.

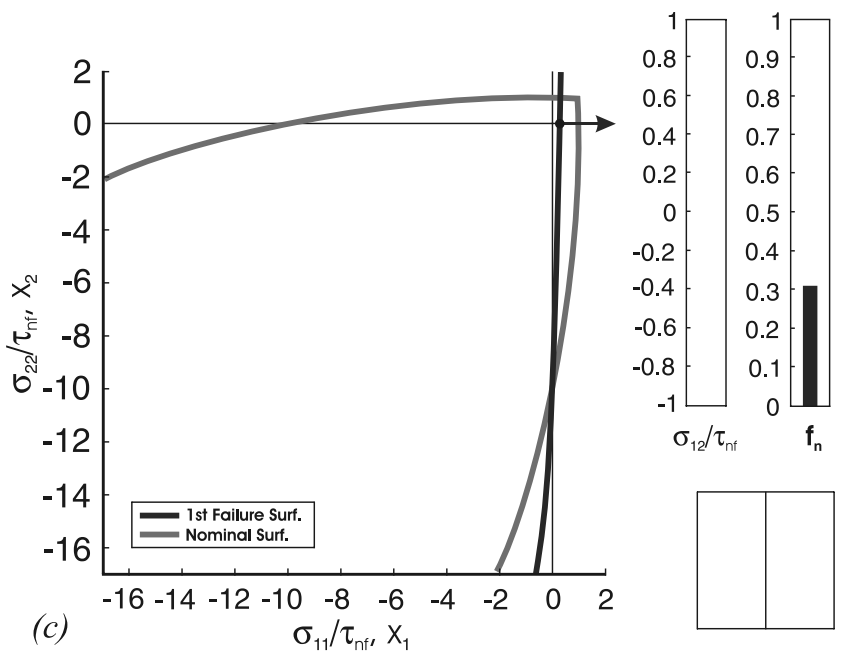

Figure 8c. Failure surfaces for uniaxial tensile stress when $f_{n}=0.3$.

\subsection{Behavior With Two Possible Failure Surfaces}

[73] There is a possibility of creating two or more failure surfaces. As an illustration, suppose one specifies monotonically increasing values of $e_{11}$ with $e_{22}=-e_{11}$, which represents a path of pure shear stress (Figure 9a) until failure initiates as indicated in Figure 9b. Again, the failure surface has a normal in the direction of maximum principal stress. The microcrack evolves as shown in Figures $9 \mathrm{a}$ and $9 \mathrm{~b}$ with the softening function, $f_{n}$, and $\sigma_{11}$ decreasing to zero while $\sigma_{22}$ continues to increase in absolute value. With increased loading, the stress point moves down the $\sigma_{22}-$ axis as shown in Figure 9c toward the limiting point of $\sigma_{22}=-f_{c}^{\prime}$ at which time two other potential failure surfaces exist with normals oriented at $\theta= \pm 5^{\circ}$ if it is specified that the normals to surfaces of failure must be at least $5^{\circ}$ apart. Suppose just one of these surfaces is activated, $\theta=-5^{\circ}$, say. Then the decohesion surfaces associated with this specific orientation are shown in Figures 9c and 9d. Note that here again, the strength in compression is essentially maintained even though a second micro-crack has developed.
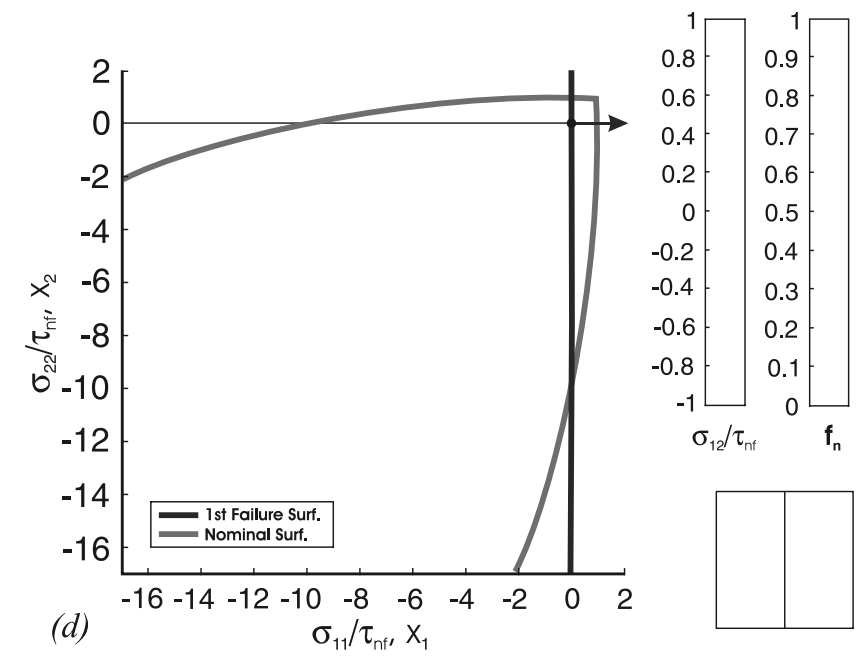

Figure 8d. Failure surfaces for uniaxial tensile stress at separation $\left(f_{n}=0\right)$. 

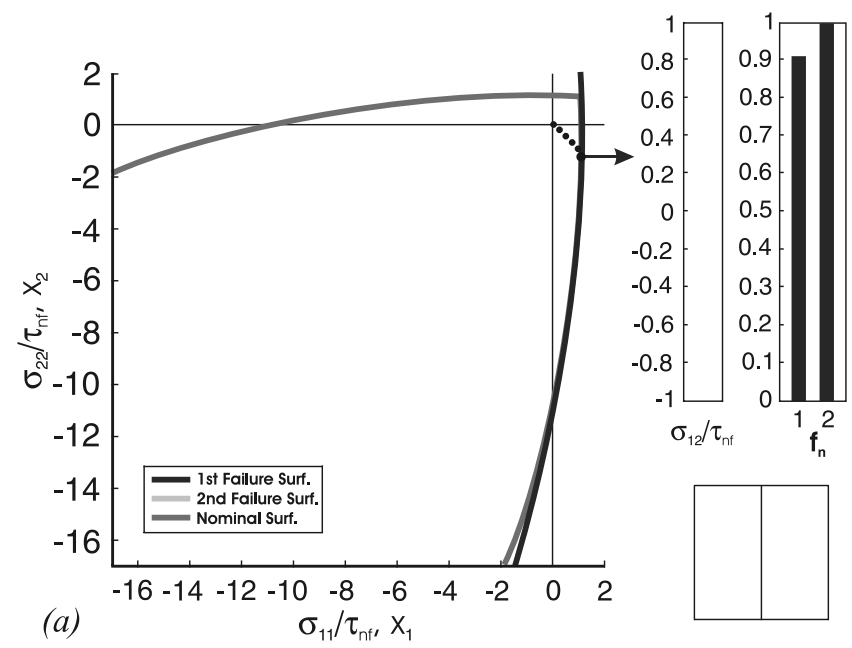

Figure 9a. The beginning of material failure $\left(f_{n 1}=0.9\right)$ for a path of pure shear in strain space.

[74] The next path is one of monotonically decreasing values of $e_{22}$ with $e_{11}=-\nu e_{22}$ which corresponds to axial compression in the $\mathrm{x}_{2}$-direction in the elastic regime. Figure 10a shows the stress path emanating from the origin and just reaching the decohesion surface. A crack initiates with $\theta=0^{\circ}$ swhich, in this case, represents axial splitting as shown in Figure 10b. Now, even if $e_{22}$ (and $\sigma_{22}$ ) are held constant, $e_{11}$ will continue to increase which indicates the crack is evolving and $f_{n}$ continues to decrease until a complete crack is formed. However, for the given strain path, the stress continues to evolve into the compressive regime and a second crack is formed as indicated in Figures 10c and 10d. Note that both the compressive and tensile strengths in the $\mathrm{x}_{2}$-direction are maintained.

\subsection{Preexisting Partial Fracture (Frozen Lead)}

[75] So far, loading paths have been considered under the assumption that the ice is initially isotropic, and anisotropy develops only when a lead is activated. An alternative and more plausible scenario for ice is the situation where failure
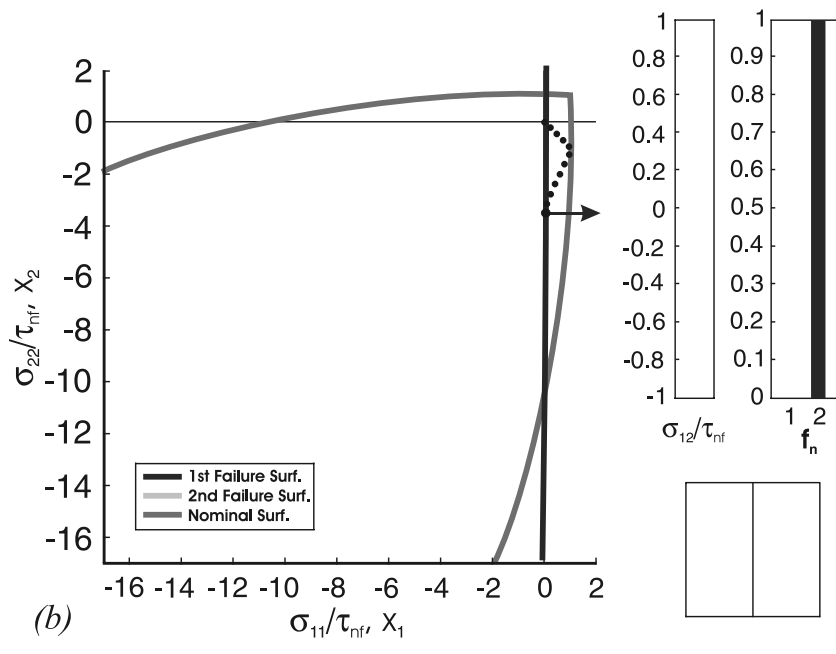

Figure 9b. The completion of the first complete crack $\left(f_{n 1}=0\right)$ for a path of pure shear in strain space.
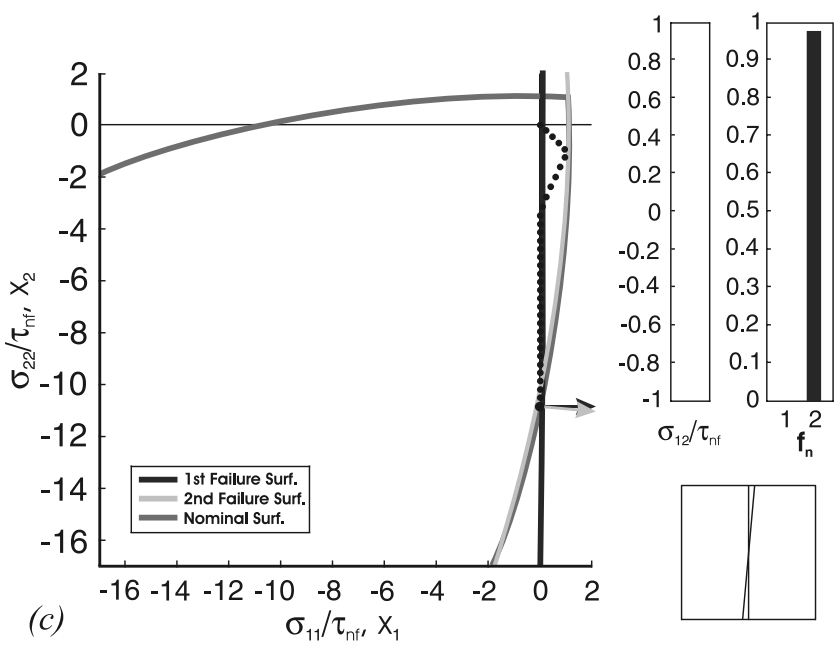

Figure 9c. The beginning of a second microcrack $\left(f_{n 2}=0.98\right)$ for a path of pure shear in strain space.

is complete as indicated by the formation of a lead, the lead has frozen, partially closed with the formation of a ridge, and frozen again but not to the extent that the strength of the original ice sheet has been fully recovered. The resulting ridge can be modeled as a partial fracture with a prescribed orientation of the normal to the ridge and an initial value selected for the softening function deemed appropriate to represent the strength of the ridge in tension. For large-scale analyses, several ridges, intersecting ridges and open leads can be constructed as initial conditions through the choice of material parameters for material points used to define the domain.

[76] To be specific, suppose an existing ridge is represented by a decohesion function associated with $f_{n}=0.3$, and an orientation of $\theta_{1}=45^{\circ}$. The corresponding complete decohesion surface is shown in Figure 11a which also shows the stress path emanating from the origin along the $\sigma_{11}-$ axis as obtained from $e_{22}=-\nu e_{11}$ with prescribed increasing values of $e_{11}$. Figure $11 \mathrm{~b}$ shows that the existing failure surface is activated, a shear stress develops, the
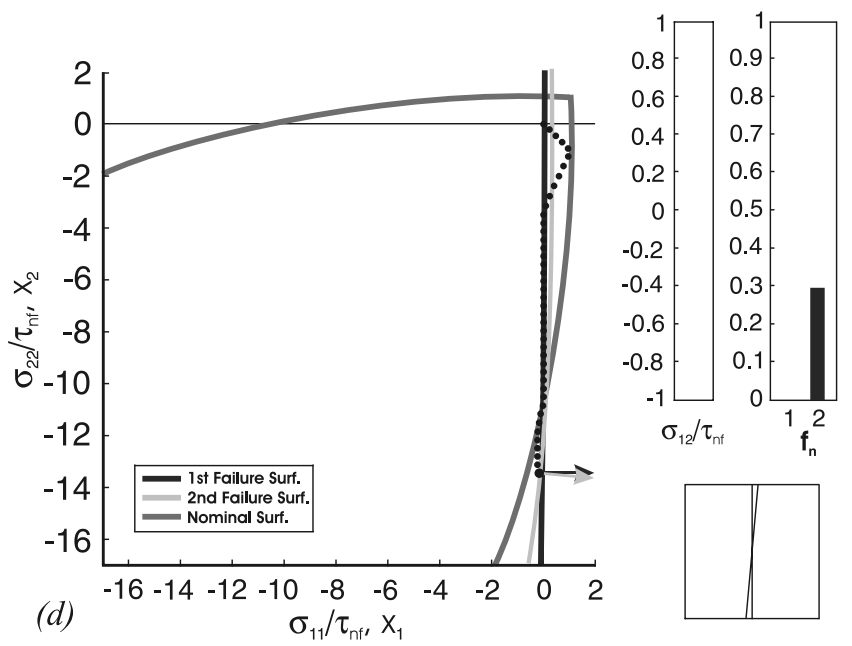

Figure 9d. The development of the second microcrack $\left(f_{n 2}=0.29\right)$ for a path of pure shear in strain space. 

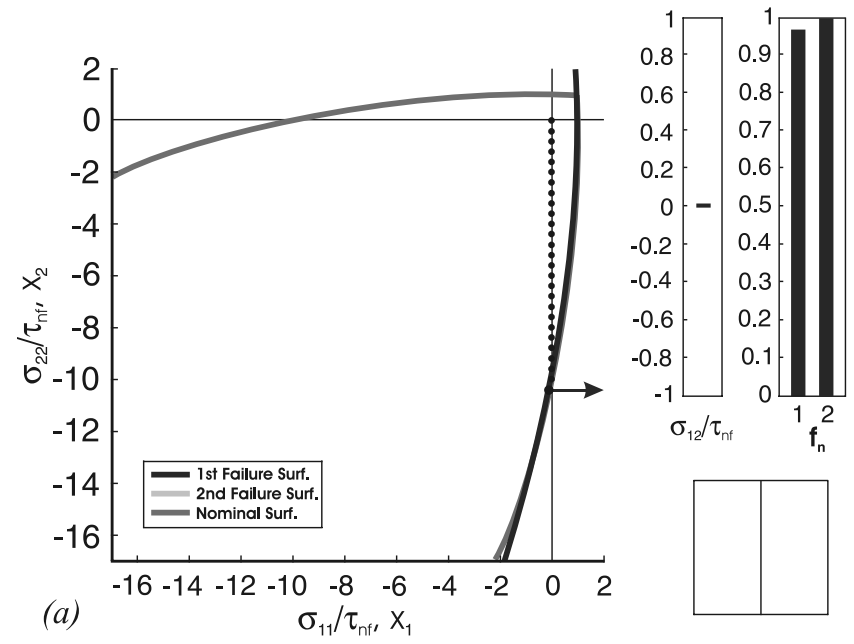

Figure 10a. Initiation of axial splitting $\left(f_{n 1}=0.97\right)$ under uniaxial compression in stress.

softening function decreases, and the stress path in $\sigma_{11}-\sigma_{22}$ space branches down from the uniaxial path. Eventually, another branch of the decohesion surface is activated (Figure 11c) and a second microcrack is initiated. Note that the orientation of the second microcrack is not $\theta_{2}=$ $0^{\circ}$ because of the presence of shear stress caused by the first microcrack. For this strain path, the first crack deactivates and the second crack evolves (Figure 11d) until failure is complete.

[77] It can be shown that a slightly different initial condition of $\theta_{1}=30^{\circ}$ and the same loading path, only the first crack will govern and a second crack will not appear. Such results show the sensitivity of the prediction of crack initiation to slight changes in initial conditions and in the direction of a stress or strain path.

\subsection{Alternative Method for Selecting Values for Material Parameters}

[78] With the previous development, there is now an alternative approach for estimating values of material
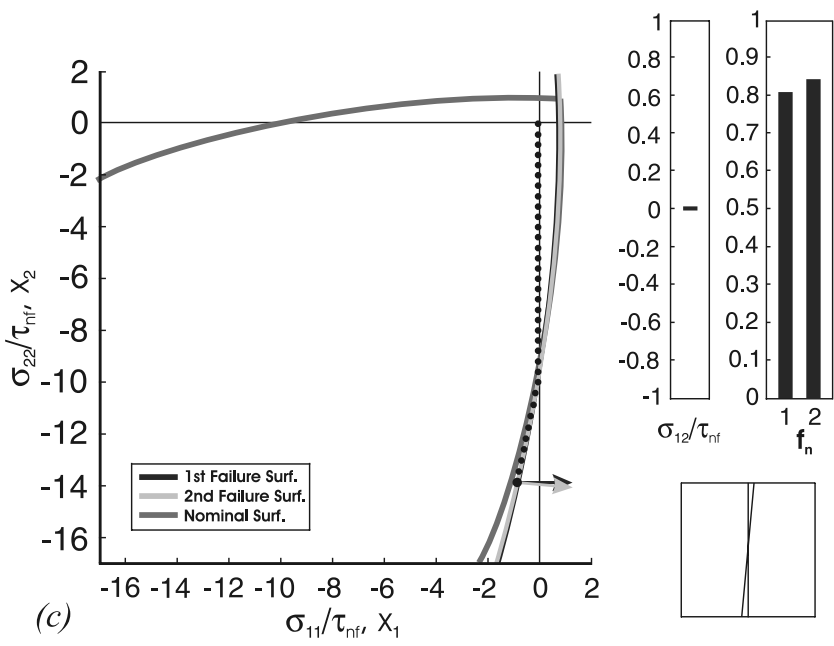

Figure 10c. Growth of two microcracks $\left(f_{n 1}=0.8\right.$ and $\left.\mathrm{f}_{\mathrm{n} 2}=0.84\right)$ as the path diverts from uniaxial compression in stress.

parameters for pack ice and that is to use the measured motion of grid points as given by satellite data for large regions of Arctic ice. The procedure is described by Kwok [1998] and data are available for several years. For this study, a $50 \mathrm{~km} \times 50 \mathrm{~km}$ region has been identified and the location of material points within the ice obtained from two consecutive satellite images 18.5 hours apart on day 136 of 2002. This motion has been decomposed under the assumption that all deformation is due to displacement discontinuity in Coon et al. [2006]. This procedure gives an optimal jump in displacement for the given deformation of an element. The jump is optimal in the sense that it gives the best fit to the observed strain. If it is believed that these displacement discontinuities are representative of what is actually observed, then when the same nodal displacements are used to describe element deformations for the elasticdecohesion model, the resulting crack deformation should
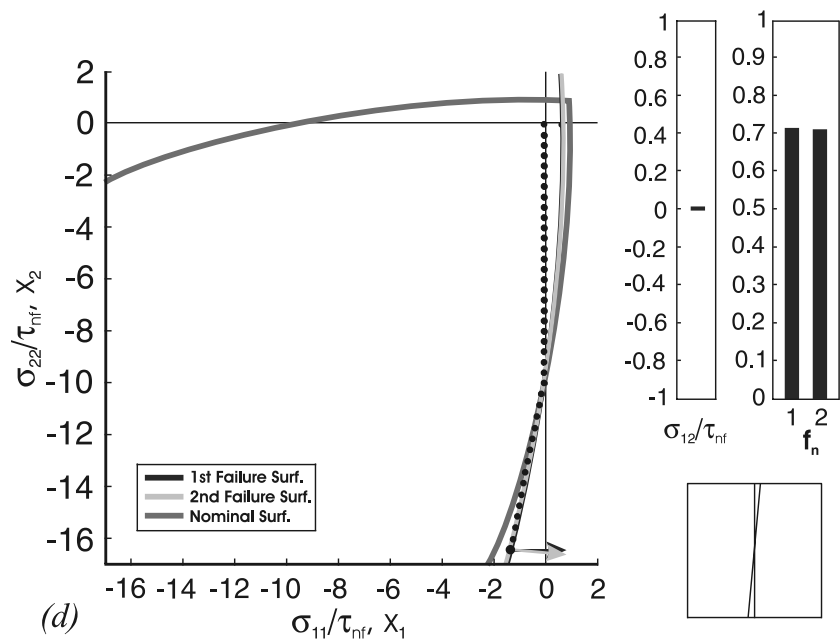

Figure 10d. Continued growth of two microcracks $\left(f_{n 1}=0.71\right.$ and $\left.f_{n 2}=0.69\right)$ as the path diverts further from uniaxial compression in stress.

Figure 10b. Initiation of a second microcrack $\left(f_{n 2}=0.98\right)$ under uniaxial compression in stress.

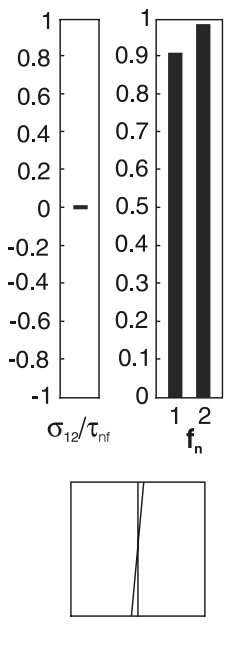

16 of 21 


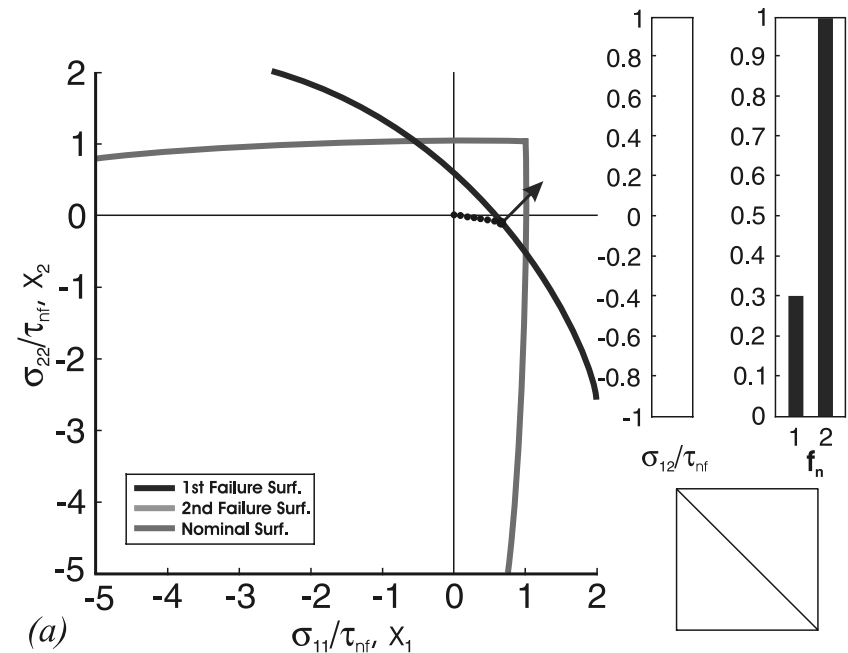

Figure 11a. Initial loading with a frozen ridge modeled as an existing microcrack with reduced strength $\left(f_{n 1}=0.3\right)$.

not be altered significantly when the elastic-decohesion constitutive equation is used.

[79] Figure 12 shows the predictions of crack displacements with the use of the constitutive equation for a limited range of material parameters. Only those cracks with $\left[u_{n}\right]^{2}+$ $\left[u_{t}\right]^{2}>u_{0}^{2}$ and $u_{0}=200 \mathrm{~m}$ are shown. Within each element, a solid bar is meant to indicate a crack, with the width of the bar proportional to the amount of normal displacement discontinuity. Shear is indicated by the relative translations of the opposite sides of the bar. The first point to make is that results can be vastly different for different choices of material parameters. The second point is that some results look more "reasonable" than others although a definitive error metric is necessary to say any one result is better than another. After some iteration on the values of material parameters, the tensile and compressive strengths of $\tau_{n f}=$ $20 \mathrm{kPa}$ and $\mathrm{f}^{\prime}{ }_{\mathrm{c}}=100 \mathrm{kPa}$ were selected. These values are the same as those presented in section 2.6. If the results

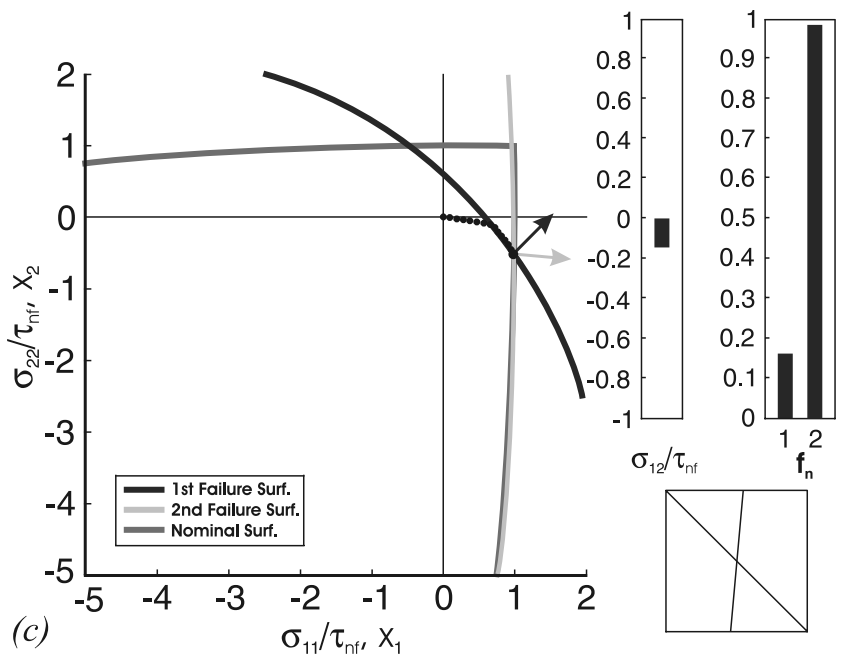

Figure 11c. Continued loading of a frozen ridge modeled as an existing microcrack $\left(\mathrm{f}_{\mathrm{n} 1}=0.16\right)$ with the initiation of a second microcrack $\left(f_{\mathrm{n} 2}=0.98\right)$.

shown in Figure 12a are assumed to be the "best" in comparison with those of Coon et al. [2006], then the shear parameter is considerably smaller than that proposed in section 2.6. In light of the complex nature of the ice pack, such uncertainty might be expected. One other important aspect not considered is that the initial condition for the ice in the analysis shown in Figure 12 is assumed to be undamaged. It is quite possible that if initial values for the orientation and the amount of softening for each element could be determined, then the larger value for the shear parameter obtained in section 2.6 might still be appropriate.

[80] In general, the effect of the initial conditions for ice on predictions for any large-scale analysis is an important subject matter that is rarely discussed, and will not be pursued here. Another significant aspect of physical behavior not included in the model is the closing of frozen leads

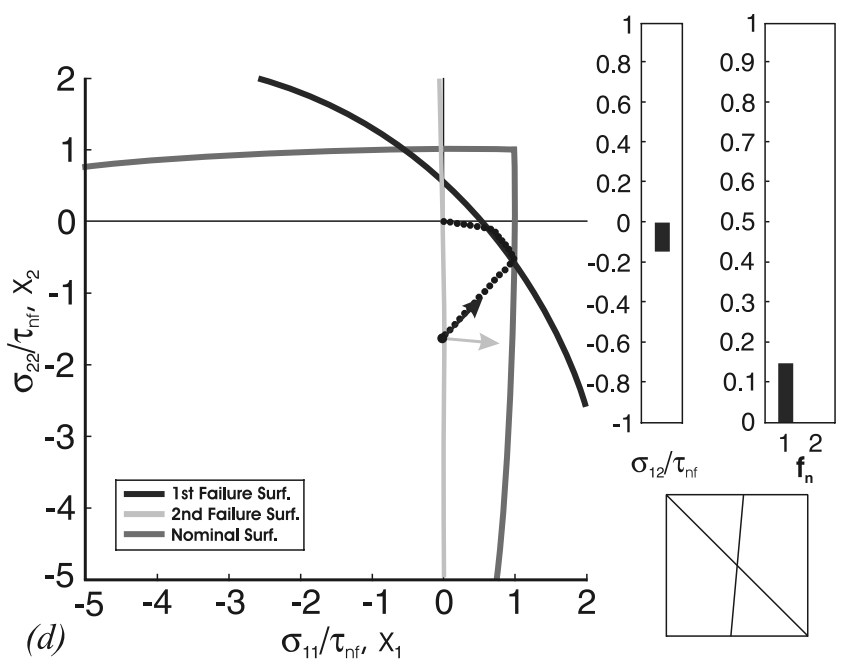

Figure 11d. Continued loading of a frozen ridge modeled as an existing microcrack $\left(f_{n 1}=0.15\right)$ with the formation of a new lead $\left(\mathrm{f}_{\mathrm{n} 2}=0\right)$.
Figure 11b. Continued loading with the initiation of failure of a frozen ridge modeled as an existing microcrack $\left(f_{n 1}=0.2\right)$. 


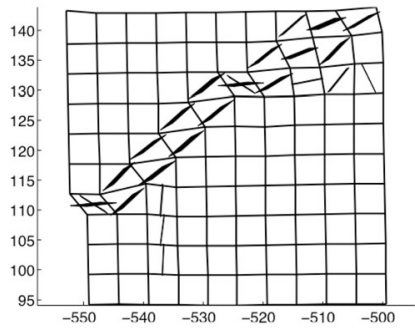

(a) $\tau_{\mathrm{sf}}=10 \mathrm{kPa}$

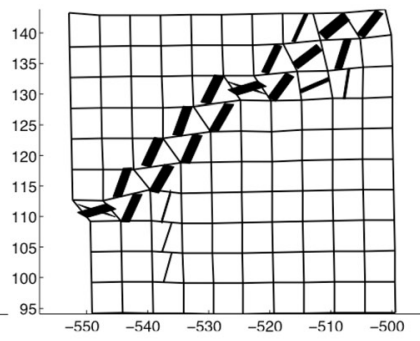

(b) $\tau_{\text {sf }}=20 \mathrm{kPa}$

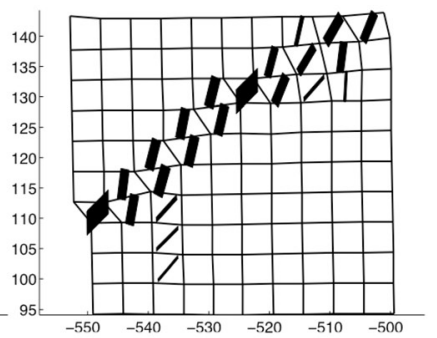

(c) $\tau_{\mathrm{sf}}=40 \mathrm{kPa}$

Figure 12. Constitutive prediction of crack opening for $\tau_{n f}=20 \mathrm{kPa}$ and $\mathrm{f}_{\mathrm{c}}^{\prime}=100 \mathrm{kPa}$.

whereby ridges are formed. This is the topic of the next section.

\section{Closure and Shear of a Frozen Lead 5.1. Initial Condition}

[81] The situation being considered is one where a lead has been formed, the lead has opened a distance, $u_{L}$, and ice motion has henceforth ceased allowing for new ice to be formed within the lead to a depth $h_{f}$. For the purpose of this study, the details of freezing including freezing rate are not considered. An idealized sketch of the geometry of the lead is shown in Figure 13a.

[82] If external forces cause the lead to close, the fresh ice may split in uniaxial compression, buckle, and break into segments that slide over and under each other to eventually form a thick conglomerate of blocks of ice called a ridge. The ridge consists of an observable feature, the sail, and a much larger segment underneath, the keel, as represented by the sketch of Figure 13b. The details of the shape of the ridge will also not be considered except to note that the total quantity of fresh ice per unit length of lead is approximately equal to the product of the width of the lead, $u_{L}$, and the depth of new or fresh ice, $h_{f}$.

[83] With time, this ridge of ice blocks will freeze and fuse together. Since a parallel set of independent leads is rarely, if ever, observed, it is reasonable to assume that the fused ice within a lead will not be as strong in tension and shear as the adjacent pack ice during the first year. The representation of the ridge as a line of weakened material was the motivation for considering a "preexisting partial fracture" in section 4.5 .

[84] An appropriate value for the tensile strength of a ridge is difficult to estimate. If the ridge is actively moving by opening, closing or shearing, the tensile strength will be zero. Only if a ridge is inactive for some time can a tensile strength develop. The object of this section is to provide an addition to the discrete constitutive model that will represent in an approximate manner the compressive stress that arises when a frozen lead closes.

\subsection{Compressive Stress With Closure of a Lead}

\subsubsection{Observed Features}

[85] Once a lead of width $u_{L}$ has formed, it is convenient to define a dimensionless parameter, $\bar{u}_{c}$, as follows:

$$
\bar{u}_{c}=1-\frac{\left[u_{n}\right]}{u_{L}} \quad 0 \leq \overline{\mathrm{u}}_{\mathrm{c}} \leq 1
$$

When the lead has formed and is stationary, then the initial value of this parameter is $\bar{u}_{c}=0$. As the lead closes, $\bar{u}_{c}$ increases. Complete closure is identified with $\bar{u}_{c}=1$ which represents an idealized condition that can only be attained if no fresh ice is formed.

[86] Both experimental data and simulations based on discrete elements [Hopkins, 1998] suggest that the closure force is rather jagged as represented symbolically by the dotted line in Figure 14. Initially, a rather large stress is necessary to start closure and this is thought to be analogous to a buckling stress. Because ice is brittle, once buckling begins, a portion of the ice breaks into segments. Subsequently, these segments ride over and under the remaining freshly frozen ice and cause further segments to break off and pile up, as reflected by the subsequent peaks and drops in stress with the peaks substantially smaller than the initial one. Ultimately, a ridge is formed at a level of stress that must be less than the failure stress in uniaxial compression, $f_{c}^{\prime}$, since a new lead orthogonal to the first is rarely observed.

[87] It is customary to approximate the observed closing stress as a function of closing displacement with a smooth

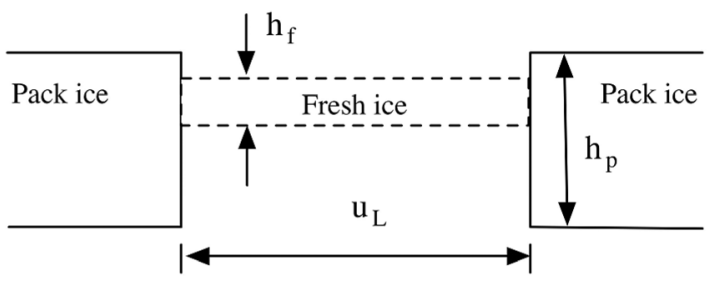

(a) Freezing of a lead

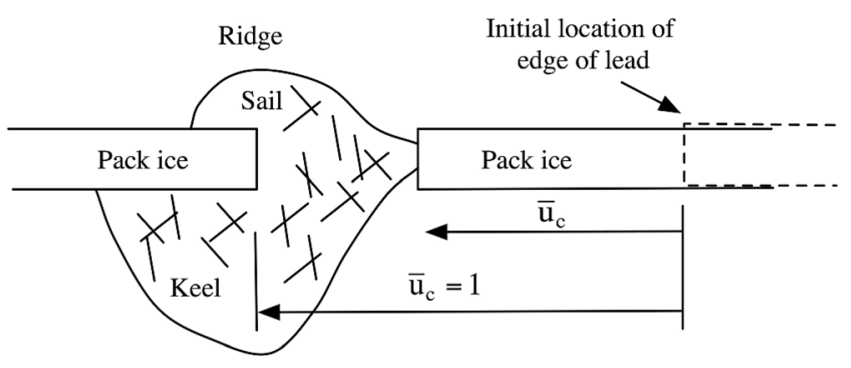

(b) Formation of a pressure ridge.

Figure 13. Idealized geometry of a frozen lead with subsequent ridge formation. 


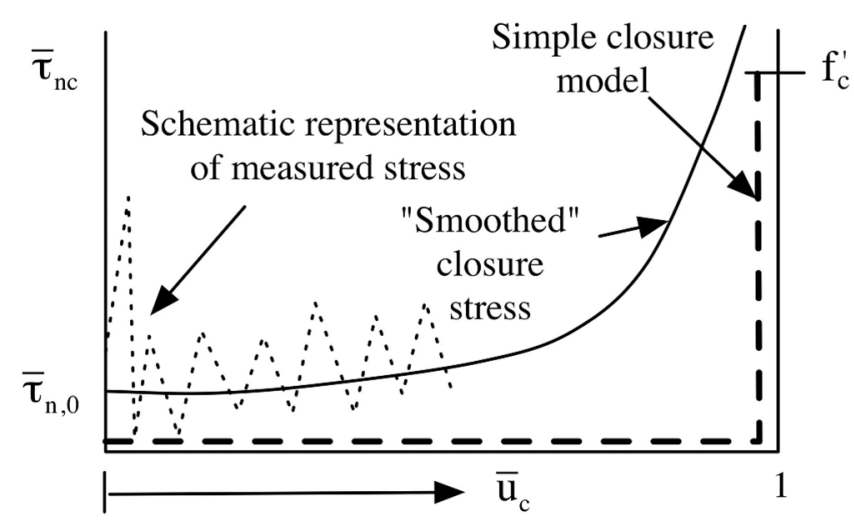

Figure 14. Various representations of stress with lead closure.

function that has an initial value $\bar{\tau}_{n c, 0}$. Typically, data indicate that the closing stress is a slowly increasing function but, ultimately, the closing force must become large to prevent one face of a lead from interpenetrating or overlapping the opposite face.

[88] Several estimates have been made for the value of the initial closing stress, all of which are of the form

$$
\bar{\tau}_{n c, 0}=\bar{\tau}_{n c, r e f}\left(h_{f} / h_{p}\right)^{\eta}
$$

where values for $\eta$ range from 1 for an argument based on crushing or shear rupture [Wilchinsky and Feltham, 2004], 3/2 if the ice is assumed to fail by buckling [Parmeter, 1974], to 2 based on gravitational potential energy within ridges [Rothrock, 1975]. The term $\bar{\tau}_{n c, \text { ref }}$ denotes a reference stress.

[89] In order to fit in smoothly with the decohesion model, both stress parameters in (53) should be considered as the total force per unit length of lead divided by the depth of the pack ice, $h_{p}$. If $\bar{\tau}_{n c, r e f}$ is not available, a simple estimate for $\bar{\tau}_{n c, 0}$ is obtained by scaling the strength of ice in compression, $f_{c}^{\prime}$, with the relative thicknesses of lead ice and pack ice:

$$
\bar{\tau}_{n c, 0}=f_{c}^{\prime} \frac{h_{f}}{h_{p}}
$$

[90] As an example, in situ stresses were measured by Coon et al. [1989] for lead ice of thickness $h_{n}=40 \mathrm{~cm}$ and a pack ice thickness of $h_{p}=160 \mathrm{~cm}$. If the value $f^{\prime}{ }_{c}=100 \mathrm{kPa}$ suggested in section 2.6 is used in (54), the result is a predicted peak compressive stress in the pack ice for lead closure of $\bar{\tau}_{n c, 0}=25 \mathrm{kPa}$, which is roughly the peak stress given by Coon et al. [1989].

[91] Based on a series of numerical simulations of lead forces averaged over a sufficiently long lead, Hopkins [1998] suggests a force of $95\left(h_{f}\right)^{3 / 2} \mathrm{kN} / \mathrm{m}$ with $h_{f}$ in meters. For $h_{n}=0.4 \mathrm{~m}$, and dividing the result by the thickness of pack ice $h_{p}=1.6 \mathrm{~m}$, Hopkins' formulation translates to an equivalent initial closing stress of $\bar{\tau}_{n c, 0}=15 \mathrm{kPa}$ on the pack ice. This value is remarkably similar to the $25 \mathrm{kPa}$ deduced above considering the widely different approaches. 5.2.2. A Simple Model

[92] The initial average closure stress is of the order of the tensile strength of the pack ice, and considerably smaller than the compressive failure stress. Therefore, one might reasonably adopt the simple representation indicated by the heavy dashed line in Figure 14 of ignoring the closure stress altogether. With this approach the effect of fresh ice is ignored as far as the stress and motion analysis is concerned. Once a lead forms, the traction is zero until the two surfaces of the lead come together at which time the material assumes the behavior of pack ice in compression with zero tensile strength in the direction normal to the lead. Such behavior is implied if the decohesion model is used with no modifications made for freezing and ridge behavior. Explicitly, if a lead is formed, the decohesion function assumes the final anisotropic form displayed for the uniaxial tensile case of Figure 8d. The decohesion surface does not change if the edges of a lead drift apart. However, the distance between the lead surfaces is known through the displacement discontinuity $\left[u_{n}\right]$. The constitutive equation for the lead reduces to that of enforcing zero traction as long as $\left[u_{n}\right]>0$, a condition that is enforced until $\left[u_{n}\right]$ reduces to zero (or $\left.\bar{u}_{c}=1\right)$. Then the zero traction condition is dropped, the elasticity constitutive equation is invoked and the decohesion condition is checked to determine if an additional lead is formed at some angle relative to the existing lead.

[93] It is possible for a number of simulations that such a simple model may be perfectly adequate. However, if the closure stress is considered to be significant, then a modification must be made to the decohesion formulation.

\subsubsection{A More Complex Model}

[94] Here, an addition to the model is proposed to provide the current strength of the fresh ice in compression. No attempt is made to represent the details of the observed abrupt increases and decreases in stress that accompany buckling and breaking of fresh ice into segments. Rather, an "average smoothed" stress is predicted that will, hopefully, be adequate for large-scale numerical simulations of Arctic ice in which some of the details of lead closure are included.

[95] Suppose a lead has been formed $\left(\left[u_{n}\right]>u_{0}\right)$ and identified with a unit normal vector, n. An additional decohesion function for lead closure and ridge formation is defined for that orientation as follows:

$$
F_{R}=-\tau_{n}-\bar{\tau}_{n c}
$$

As before, $F_{R}>0$ is not allowed, $F_{R}<0$ implies that the response is elastic and $F_{R}=0$ or $\tau_{n}=-\bar{\tau}_{n c}$ is identified with closing. The current strength of the fresh ice in compression is identified with $\bar{\tau}_{n c}$, which is always positive, so closure can be activated only if $\tau_{n}$ is negative and sufficiently large.

[96] Suppose the current strength can be represented through the use of an exponential function:

$$
\bar{\tau}_{n c}=\bar{\tau}_{n, 0} e^{\zeta \bar{u}_{c}}
$$

in which $\bar{\tau}_{n c, 0}$ denotes the initial strength of fresh ice given by (53). The parameter, $\zeta$, in (56), provides the rate at which strength increases with closure and, for the moment, its value is considered to be chosen empirically based on in situ evidence. In particular, a value for $\zeta$ must be selected to ensure that full strength in compression is achieved prior to 
complete closure $\left(\bar{u}_{c}=1\right)$ to prevent a prediction that the faces of the lead will "overlap".

[97] If an associated evolution equation for closure is adopted, then

$$
\left[\dot{u}_{n}\right]=\dot{\omega}_{c} u_{L} \frac{\partial F_{R}}{\partial \tau_{n}}
$$

in which $u_{L}$ is included to render the monotonically increasing closing parameter $\omega_{c}$ dimensionless. The result of combining (57) and the rate form of (52) is that

$$
\omega_{c}=\bar{u}_{c} \quad\left[\dot{u}_{n}\right]=-\dot{\bar{u}}_{c} u_{L}
$$

The consistency condition, $\dot{F}_{R}=0$, merely yields $\tau_{n}=-\bar{\tau}_{n c}$ in rate form. At this stage, only closing of the lead is considered. Further modifications would be necessary to handle the situation where closure is stopped, perhaps the lead opens again, further freezing occurs and then the lead resumes closing.

[98] To illustrate the model in more detail, Figure 15 shows various surfaces and paths in the space of traction components $\tau_{n}$ and $\tau_{\mathrm{t}}$. The decohesion surface, $F_{n}=0$, is given by the line A-B-C with the softening function assigned a value $f_{n}=0$ which corresponds to the complete formation of a lead. With freezing some tensile strength may be recaptured and this recapture can be reflected by designating a small, but nonzero, value for $f_{n}$. However, once closure begins there is no tensile strength so, for convenience, $f_{n}$ is fixed at zero.

[99] Once some new ice is formed, a compressive strength is introduced through the use of the ridging function. The corresponding surface in the space of $\tau_{n}$ and $\tau_{\mathrm{t}}$ is $F_{R}=0$ and designated by line D-E-F in Figure 15. Lead closure is reflected in the same space by a traction path that goes from the origin to point $\mathrm{E}$, an increase in the normal component of traction until the initial value, $\bar{\tau}_{n c, 0}$, of the smooth form of the ice crushing strength is reached. With closure, the ridging surface moves to the left with the traction point moving to point $\mathrm{E}^{\prime}$. The new decohesion surface for ridging is line $\mathrm{D}^{\prime}-\mathrm{E}^{\prime}-\mathrm{F}^{\prime}$, and the evolution equation provides the exponential form of the stress versus lead closure relation shown in Figure 14.

\subsection{Modeling the Motion of a Lead in Shear}

[100] Often the linear features displayed by Kwok [1998] are accompanied by large measures of shear. Expressed another way, it is not unusual for leads to be accompanied by large relative tangential motions. Here, it is argued that the existing model for decohesion, when combined with the ridging formulation can also provide a representation for shear motion.

[101] Photographs of leads often show that leads are not straight but are jagged. Therefore, even after a lead has formed, a significant shear stress is necessary to overcome the surface asperities. When shear motion is initiated the sawtooth shape of the lead will tend to force the lead surfaces apart and increase the average normal compressive traction component, $\tau_{n}$. A possible path in the space of $\tau_{n}$ and $\tau_{\mathrm{t}}$ is that designated B-G-H in Figure 15. Until the initial ridge closure stress $\bar{\tau}_{n c, 0}$ is reached, the associated flow rule indicates that $\left[\dot{u}_{n}\right]$ is positive and, hence, opening

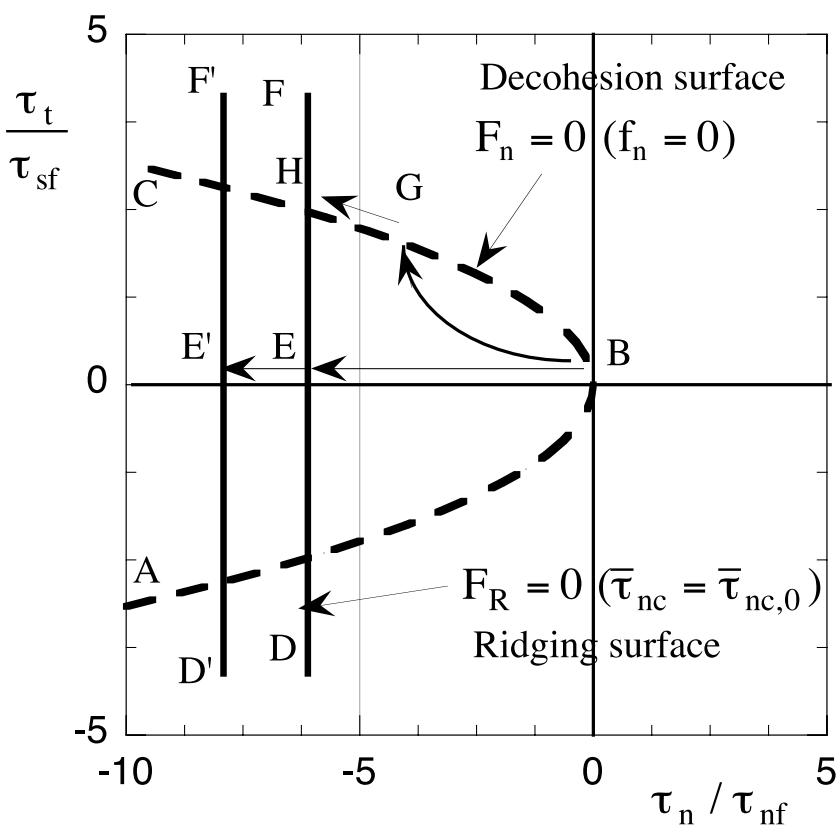

Figure 15. Decohesion surface for ridging and possible path involving shear.

continues to occur. However, when the traction moves to the corner of the two surfaces representing both decohesion and closure, it is possible to have the condition $\left[\dot{u}_{n}\right]=0$. This equation implies that no additional opening is occurring and the deformation is pure tangential motion. Alternatively, it is entirely feasible to invoke more sophisticated models from other areas of crack analysis such as the one described by Carol et al. [1997].

[102] It should be mentioned that the essential ideas reflected in the composite decohesion and closing surface of Figure 15 have been suggested much earlier by Coon et al. [1992, 1998] and Pritchard [1998].

\section{Summary}

[103] A decohesive approach has been proposed for modeling the initiation and evolution of leads within the Arctic ice pack which otherwise can be represented as an elastic continuum. Such an approach is different from the more common procedure of using a continuum constitutive equation with a failure criterion to simulate leads, or from modeling the complete ice pack as discrete floes. The decohesive model offers advantages in that interpretation of material parameters is simpler, the basic variables of the formulation such as normal and tangential components of lead-displacement discontinuity are more in tune with observations, and weaknesses associated with previous leads can be easily accommodated.

[104] Important features of the model include the following: (1) A lead can initiate and evolve even if the stress is one of uniaxial compression. (2) The orientation of the lead and the mode of failure depend on the state of stress. (3) Lead branching can be predicted. (4) A variety of preexisting lines of weakness can be accommodated through the choice of initial values for material parameters. 
[105] With regard to lead closure, an elementary addition to the model provides a method for predicting stress resultants during ridge formation and shearing motion. However, there are numerous issues that have not been included. Specifically no attempt has been made to include ice freezing and thawing, and multiple sequences of opening and closing of leads with ice redistribution.

[106] In conclusion, it is believed that the ability to specifically model the failure of ice as reflected by the orientation and location of leads, lead opening, lead closing and large shear motion represents a significant enhancement to existing approaches. As indicated by preliminary analyses performed by Sulsky et al. [2006], numerical solutions to boundary value problems can be obtained at a computational cost comparable to the most efficient methods being used currently.

[107] Acknowledgment. The financial support of the National Science Foundation (under grant DMS-0222253), the Minerals Management Service and the National Aeronautics and Space Administration (under contract NNH04 CC 45C), and the Office of Naval Research (under contract N000-14-04-M-0005) is gratefully acknowledged.

\section{References}

Alfaiate, J., A. Simone, and L. J. Sluys (2003), Non-homogeneous displacement jumps in strong embedded discontinuities, Int. J. Solids Struct., $40,5799-5817$

Bazant, Z. P. (2002), Scaling of sea ice fracture-Part I: Vertical Penetration, J. Appl. Mech., 69, 11-18.

Carol, I., P. C. Prat, and C. M. Lopez (1997), Normal/Shear Cracking Model: Application to Discrete Crack analysis, J. Eng. Mech., 123(8), $765-773$.

Coon, M. D., G. A. Maykut, R. S. Pritchard, D. A. Rothrock, and A. S. Thorndike (1974), Modeling the pack sea ice as an elastic-plastic material, AIDJEX Bull., 24, 1-105.

Coon, M. D., P. A. Lau, S. H. Bailey, and B. J. Taylor (1989), Observations of ice floe stresses in the eastern Arctic, paper presented at Conference on Port and Ocean Engineering Under Arctic Conditions (POAC '89), Lulea Univ. of Technol., Lulea, Sweden.

Coon, M. D., D. C. Echert, and G. S. Knoke (1992), Pack ice anisotropic constitutive law, paper presented at 11th International Symposium on Ice, Int. Assoc. for Hydraul. Res., Banff, Alberta, Canada.

Coon, M. D., G. S. Knoke, D. C. Echert, and R. S. Pritchard (1998), The architecture of an anisotropic elastic-plastic sea ice mechanics constitutive law, J. Geophys. Res., 103, 21,915-21,925.

Coon, M. C., D. C. Echert, and G. S. Knoke (1999), Stress validation of a failure (yield) surface for pack ice, in Ice in Surface Waters, edited by H. T. Shen, pp. 1049-1055, A. A. Balkema, Brookfield, Vt.

Coon, M. D., R. Kwok, G. Levy, M. Pruis, H. Schreyer, and D. Sulsky (2006), AIDJEX assumptions revisited and found inadequate, J. Geophys. Res., doi:10.1029/2005JC003393, in press.

Hibler, W. D. (1979), A dynamic thermodynamic sea ice model, J. Phys. Oceanogr., 9, 815-846.

Hibler, W. D. (2001), Sea ice fracturing on the larger scale, Eng. Fract. Mech., 68, 2013-2043.

Hibler, W. D., and E. M. Schulson (1997), On modeling sea ice fracture and flow in numerical investigation of climate, Ann. Glaciol., 25, 26-32.

Hibler, W. D., and E. M. Schulson (2000), On modeling the anisotropic failure and flow of flawed sea ice, J. Geophys. Res., 105, 17,105-17,120.

Hopkins, M. A. (1996), On the mesoscale interaction of lead ice and floes, J. Geophys. Res., 101, 18,315-18,326.

Hopkins, M. A. (1998), Four stages of pressure ridging, J. Geophys. Res., $103,21,883-21,891$.

Hopkins, M. A. (2001), Effect of tensile strength in the Arctic pack, in Proceedings of IUTAM Symposium on Scaling Laws in Ice Dynamics, edited by J. P. Dempsey and H. H. Shen, pp. 373-386, Springer, New York.

Hunke, E. C. (2001), Viscous-Plastic Sea Ice Dynamics with the EVP Model: Linearization Issues, J. Comp. Phys., 170, 18-38.
Hunke, E. C., and K. Dukowicz (1997), An elastic-viscous-plastic model for sea ice dynamics, J. Phys. Oceanogr., 27, 1849-1867.

Koerner, R. M. (1973), The mass balance of the sea ice of the Arctic Ocean, J. Glaciol, , 12, 173-185.

Kwok, R. (1998), The RADARSAT geophysical processor system, in Analysis of SAR Data of the Polar Oceans: Recent Advances, edited by C. Tsatsoulis and R. Kwok, pp. 235-257, Springer, New York.

Kwok, R. (2006), Contrasts in Arctic Ocean sea ice deformation and production in the seasonal and perennial ice zones, J. Geophys. Res., 111, C11S22, doi:10.1029/2005JC003246.

Lade, P. V. (2001), Modeling sea ice as an elasto-plastic frictional material, in IUTAM Symposium on Scaling Laws in Ice Mechanics and Ice Dynamics, edited by J. P. Dempsey and H. H. Shen, pp. 227-238, Springer, New York.

Larsson, R., and K. Runesson (1996), Element-based localization band based on regularized displacement discontinuity, J. Eng. Mech., 122, $402-411$.

Moës, N., J. Dolbow, and T. Belytschko (1999), A finite element method for crack growth without remeshing, Int. J. Numer. Methods Eng., 46, $131-150$.

Parmeter, R. R. (1974), A mechanical model of rafting, AIDJEX Bull., 23, 97-115.

Planas, J., M. Elices, G. V. Guinea, F. J. Gómez, D. A. Cendón, and I. Arbilla (2003), Generalizations and specializations of cohesive crack models, Eng. Fract. Mech., 70, 1759-1776.

Pritchard, R. S. (1998), Ice conditions in an anisotropic sea ice dynamics model, Int. J. Offshore Polar Eng., 8, 9-15.

Rashid, Y. R. (1968), Analysis of prestressed concrete pressure vessels, Nucl. Eng. Design, 7(4), 334-355.

Renshaw, C. E., and E. M. Schulson (2001), Universal behavior in compressive failure of brittle materials, Nature, 412, 897-900.

Rothrock, D. A. (1975), The energetics of the plastic deformation of pack ice by ridging, J. Geophys. Res., 80, 4514-4519.

Sammonds, P. R., S. A. F. Murrell, and M. A. Rist (1998), Fracture of multiyear sea ice, J. Geophys. Res., 103, 21,795-21,815.

Schreyer, H. L. (2001), Modeling failure initiation in sea ice based on loss of ellipticity, in Proceedings of IUTAM Symposium on Scaling Laws in Ice Mechanics and Ice Dynamics, edited by J. P. Dempsey and $\mathrm{H}$. H. Shen, pp. 239-250, Springer, New York.

Schreyer, H. L., D. L. Sulsky, and S.-J. Zhou (2002), Modeling delamination as a strong discontinuity with the material point method, Comput. Methods Appl. Mech. Eng., 191, 2483-2508.

Schulson, E. M. (2001), Brittle failure of ice, Eng. Fract. Mech., 68, 18391887.

Schulson, E. M. (2004), Compressive shear faults within Arctic sea ice: Fracture on scales large and small, J. Geophys. Res., 109, C07016, doi:10.1029/2003JC002108.

Sulsky, D., H. Schreyer, K. Peterson, R. Kwok, and M. Coon (2006), Using the material-point method to model sea ice dynamics, J. Geophys. Res., doi:10.1029/2005JC003329, in press.

Thorndike, A. S., D. S. Rothrock, G. A. Mykut, and R. Colony (1975), The thickness distribution of sea ice, J. Geophys. Res., 80, 4501-4513.

Wells, G. N., and L. J. Sluys (2001a), Analysis of slip planes in threedimensional solids, Comput. Methods Appl. Mech. Eng., 190, 35913606.

Wells, G. N., and L. J. Sluys (2001b), A new method for modelling cohesive cracks using finite elements, Int. J. Numer. Methods Eng., 190, $2667-2682$.

Wells, G. N., and L. J. Sluys (2001c), Three-dimensional embedded discontinuity model for brittle fracture, Int. J. Solids Struct., 138 , $897-913$.

Wilchinsky, A. V., and D. L. Feltham (2004), A continuum anisotropic model of sea-ice dynamics, Proc. R. Soc. London. A, 460, 2105-2140.

Zubov, N. N. (1943), Arctic Ice, Izdatel'stvo Glasevmorputi, Moscow. (English translation, $A D 426972$, U.S. Nav. Oceanogr. Off., Stennis Space Cent., Miss.) (Available from Natl. Tech. Inf. Serv., Springfield, Va.).

M. D. Coon, Northwest Research Associates, Seattle, WA 98009, USA. R. Kwok, Jet Propulsion Laboratory, Pasadena, CA 91109, USA.

L. B. Munday and H. L. Schreyer, Department of Mechanical Engineering, University of New Mexico, Albuquerque, NM 87131, USA. (schreyer@unm.edu)

D. L. Sulsky, Department of Mathematics and Statistics, University of New Mexico, Albuquerque, NM 87131, USA. 